Iglesias y Llorente / Revista de Economía Laboral 12 (2015), 164-200

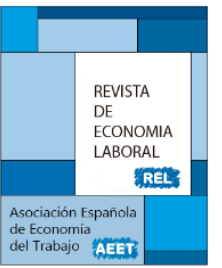

\title{
LA REFORMA LABORAL DE 2012 Y EL CRECIMIENTO DEL EMPLEO A TIEMPO PARCIAL INVOLUNTARIO. ¿NUEVOS MECANISMOS DE AJUSTE DEL MERCADO DE TRABAJO?
}

\author{
Carlos Iglesias Fernández ${ }^{1^{*}}$, Raquel Llorente Heras ${ }^{\&}$ \\ *Universidad de Alcalá, \&Universidad Autónoma de Madrid
}

\section{Resumen}

Recibido Julio 2015; Aceptado Octubre 2015

El empleo a tiempo parcial (ETP), especialmente el de carácter involuntario, ha crecido notablemente durante la crisis. Algunos trabajos vinculan esta situación con su uso empresarial como mecanismo de flexibilidad, al permitir ajustar las horas de trabajo a la evolución del ciclo. Por otra parte, la reforma laboral de 2012 ha introducido importantes mecanismos de flexibilidad interna para las empresas, especialmente en relación con el tiempo de trabajo. El objetivo del artículo será doble. Primero, determinar en qué medida están cambiando los determinantes del ETP, sustituyéndose su rol habitual como opción voluntaria de compatibilización por otro involuntario como mecanismo de flexibilización. Segundo, analizar el papel desempeñado por la reforma de 2012 en la explicación de estas tendencias. Los resultados señalan que, aunque se han producido cambios profundos en el perfil del ETP, resulta cuestionable que se fundamenten en los contenidos de la reforma laboral. Al contrario, parece que son las propias consecuencias de la crisis las responsables de estos cambios.

Palabras clave: tiempo parcial involuntario, reforma laboral, flexibilización, género. Clasificación JEL: J22, J88, J16.

\section{Abstract}

Part-time employment (PTE), especially for involuntary reason, has growth in a remarkable way during the crisis. Some literature links this situation with its use like a flexibility tool for firms, allowing adjust working time to economic cycle. Moreover, the labor reform of 2012 has introduced important mechanisms of internal flexibility for companies, especially concerning working time. Based on these ideas, the aim of the article is twofold. First, determine to what extent it is changing the determinants of PTE in Spain, replacing his usual role as a voluntary option for balance household and professional spheres for another involuntary as a mechanism for flexibility. Second, examine the role played by the labor reform of 2012 in explaining these trends. The results show that although there have been profound changes in the profile of the PTE, it is questionable that are based on the contents of the labor reform. Instead, it appears that the own consequences of the crisis are the responsible for these changes.

Key words: Involuntary part-time, Labour market reform, Flexibility, Gender. JEL Classification: J22, J88, J16.

\footnotetext{
${ }^{1}$ Autor para correspondencia: carlos.iglesias@uah.es
}

\footnotetext{
C Revista de Economía Laboral
} 


\section{Introducción}

El uso del empleo a tiempo parcial ocupa un lugar destacado dentro de la Política Laboral. Su aplicación se justifica tradicionalmente sobre dos argumentos fundamentales. En primer lugar, por su contribución a la creación de empleo - en la medida en que incrementaría tanto la oferta de trabajo, al permitir la participación laboral de ciertos colectivos excluidos si su única opción fuera el trabajo a tiempo completo - como la demanda de trabajo - ya que mejoraría la productividad del factor de trabajo al aumentar las opciones de organización de los procesos de trabajo de que dispondrían las empresas (O`Reilly, 1996). En segundo lugar, por su contribución a la promoción laboral de la mujer, al facilitar la conciliación de las esferas profesional y familiar, sobre la base de la división sexual del trabajo que tiene lugar en la familia y que habitualmente conduce a que las mujeres sean quienes se responsabilicen en mayor medida de las tareas de cuidado y crianza de los hijos (Fagan et al., 2001). Ambos argumentos conducirían a lo que podríamos denominar como una visión normalizada del empleo a tiempo parcial, definida por constituirse en una opción voluntaria ${ }^{2}$, con la que incrementar las alternativas laborales de los trabajadores, especialmente en relación con la población femenina, al mejorar sus posibilidades de compatibilización.

Recientemente, sin embargo, se asiste a un incremento del empleo a tiempo parcial de carácter involuntario (OCDE, 2010) definido como aquellos trabajadores que realizan un trabajo a tiempo parcial debido a no haber podido encontrar un trabajo a tiempo completo a pesar de desearlo. Esta situación se vincularía con su utilización como mecanismo de flexibilidad en el mercado de trabajo, al permitir a los empresarios ajustar las horas de trabajo a la evolución del ciclo económico (Buddelmeyer et al., 2008), e implicaría la observación de situaciones de subutilización del factor trabajo (Canon et al., 2014; Kjeldstad and Nymoen, 2009; Petrongolo, 2004). El resultado final sería una profunda modificación tanto de la naturaleza como de las decisiones y colectivos relacionados con este tipo específico de empleo.

Aunque los trabajos que analizan los determinantes del empleo a tiempo parcial son muy numerosos, habitualmente se centran en el

\footnotetext{
2 Es posible que estas decisiones no sean tan voluntarias, en la medida en que las mujeres las emprendan anticipando un mercado de trabajo donde recibirán un trato diferenciado, si no discriminatorio, en comparación con los hombres (Rubery et al., 1996).
} 
colectivo femenino, al entenderse tradicionalmente que este tipo de empleo está concentrado en mujeres, especialmente con poca cualificación y desempeñando ocupaciones laborales de baja cualificación del sector servicios (Petrongolo, 2004, por ejemplo). Sin embargo, la emergencia de los nuevos patrones asociados al empleo a tiempo parcial se refleja en la aparición de trabajos interesados en considerar su carácter involuntario.

Así, Cam (2012) analiza los determinantes del empleo a tiempo parcial diferenciando entre la parcialidad voluntaria e involuntaria, y considerando el género como una variable central. Este trabajo considera tres tipos fundamentales de argumentos explicativos: los demográficos (edad y estado civil) los laborales (sector de actividad, titularidad pública de la empresa, el tamaño del establecimiento y el grado de sindicación) y los vinculados con el estatus laboral (el nivel de educación poseído y la ocupación laboral desempeñada).

En Kjeldstad y Nymoen (2012) se intenta diferenciar las decisiones que conducen hasta el empleo a tiempo parcial en función de su voluntariedad o involuntariedad. En términos generales, estos autores concluyen que las variables de demanda explican mejor el trabajo a tiempo parcial involuntario, y son neutrales por género, mientras que las variables de oferta explican mejor el trabajo a tiempo parcial voluntario y sesgarían por género sus efectos, respondiendo en mayor medida a una definición femenina.

Más allá de la distinción entre trabajo a tiempo parcial voluntario o involuntario, algunos trabajos también se interesan por la relación existente entre el empleo a tiempo parcial y la dinámica y funcionamiento del mercado de trabajo, lo que se relaciona con la hipótesis de que la evolución de este tipo de empleo se vincularía con su uso como mecanismo emergente de ajuste del mercado de trabajo. En este sentido, Buddelmeyer et al. (2008) analiza los efectos del ciclo económico sobre el empleo a tiempo parcial. Por su parte, Valletta y Bengali (2013) señalan como el tiempo parcial se ha incrementado notablemente no solo en la actual crisis sino en todas las anteriores, lo que claramente apunta a su comportamiento contracíclico y, por tanto, a su utilización como mecanismo de ajuste laboral en las crisis económicas. Por último, en De Pedraza et al. (2010) se analizan los determinantes del empleo a tiempo parcial desde un punto de vista longitudinal, como forma de indagar en el efecto ejercido por variables vinculadas con la dinámica del mercado de trabajo. El hecho de cambiar de sector, el número de contratos firmados o las experiencias previas de desempleo o inactividad son algunas de las variables consideradas por estos autores. 
De acuerdo con las cifras de la Encuesta de Población Activa (EPA), el recurso al empleo a tiempo parcial dentro del contexto de destrucción de empleo introducido por la crisis económica ha experimentado un crecimiento notable, desde una tasa de parcialidad del $12,6 \%$ durante el segundo trimestre de 2005 hasta otra del 16,4\% en el segundo trimestre de 2014 (lo que acumula un crecimiento para el periodo aproximadamente del $30 \%$ ).

En el segundo trimestre de 2005 el 30,3\% del empleo parcial masculino podía catalogarse como involuntario puro, frente al 32,9\% para el caso de las mujeres. Sin embargo, en el segundo trimestre del año 2014, ambas cifras no solo se incrementan espectacularmente sino que además cambian los roles de género, siendo considerado como involuntario el $69,3 \%$ del empleo parcial para los hombres frente al $61,1 \%$ del empleo parcial de las mujeres.

Más allá de su explicación teórica, y en relación con el caso específico de España, cabe preguntarse por la existencia de alguna relación entre este incremento observado en el empleo a tiempo parcial involuntario con la reforma laboral aprobada en 2012. En este sentido debe destacarse como el Real Decreto 3/2012 de medidas urgentes para la reforma del mercado laboral ha introducido importantes modificaciones en el marco regulatorio existente sobre el funcionamiento de nuestro mercado de trabajo, entre las cuales destaca la puesta a disposición de los empresarios de mecanismos con los que incrementar la flexibilidad laboral interna de las empresas, facilitando la modificación de las condiciones laborales, especialmente en relación con el tiempo de trabajo.

Sobre la base de las anteriores ideas, el artículo se propone como objetivo evaluar la reforma normativa emprendida sobre la regulación de nuestro mercado de trabajo en 2012 a la luz de sus efectos sobre el tiempo de trabajo, aproximados mediante el empleo a tiempo parcial de carácter involuntario. La hipótesis principal que se desea verificar con este trabajo es que las modificaciones normativas introducidas han propiciado, como se proponía en sus objetivos, un cambio en el funcionamiento y ajuste del mercado de trabajo español, que se basaría ahora en mayor medida en la modificación del tiempo de trabajo y el uso del empleo a tiempo parcial a través de las posibilidades introducidas en relación con la flexibilidad interna de las empresas.

El artículo utilizará datos procedentes de la Encuesta de Población Activa, para los años comprendidos entre 2008 y 2014 en su versión tradicional, y para los años 2011 y 2013 en su versión enlazable. Con estos datos nuestro objeto de estudio será, en primer lugar, la diferenciación entre tiempo parcial y completo y, posteriormente, la 
diferenciación entre tiempo parcial voluntario e involuntario. No obstante, se ofrecerá algún pequeño apunte sobre la cuantificación de la jornada en cada modalidad o el cambio de las horas medias de trabajo.

El resto del artículo organiza sus contenidos de acuerdo con el siguiente esquema. El apartado 2 revisa y sintetiza los contenidos de la reforma laboral de 2012 relativos a la modificación de la jornada laboral y del tiempo de trabajo. Seguidamente, el apartado 3 realizar una descripción de la evolución y de las principales características del empleo a tiempo parcial de carácter involuntario. Los análisis se desarrollan en los apartados 4 (análisis shift-share de la evolución del empleo a tiempo parcial) y 5 (determinantes de trabajar a tiempo parcial general e involuntario). El artículo finaliza interpretando en el apartado 6 los resultados alcanzados en forma de conclusiones.

\section{El tiempo de trabajo en la reforma laboral de 2012}

La reforma laboral introducida en España en el año 2012 declara en su introducción que su objetivo es "establecer un marco claro que contribuya a la gestión eficaz de las relaciones laborales [apostando] por el equilibrio en la regulación de nuestras relaciones de trabajo: equilibrio entre la flexibilidad interna y la externa [mediante] un conjunto coherente de medidas que pretenden [entre otras cosas]incentivar la flexibilidad interna en la empresa como medida alternativa a la destrucción de empleo ${ }^{3}$.

Para ello, la reforma considera un buen conjunto de medidas orientadas, en su motivación, a favorecer la empleabilidad de los trabajadores (capítulo I), fomentar la contratación indefinida y la creación de empleo (capítulo II), estimular la flexibilidad interna en las empresas (capítulo III) y mejorar la eficiencia del mercado de trabajo y reducir la dualidad (capítulo IV). A los efectos de los objetivos del artículo, y aun cuando el contrato a tiempo parcial es abordado en el capítulo II, el núcleo de medidas más estrechamente relacionadas con la regulación del tiempo de trabajo se recogen en el capítulo III, dedicado a la exposición de medidas con las que favorecer la flexibilidad interna en las empresas.

\footnotetext{
${ }^{3}$ Real Decreto-ley 3/2012 de 10 de febrero, de medidas urgentes para la reforma del mercado laboral.
} 
En este sentido, cabe mencionar que el contenido del capítulo II se reduce a aprobar la posibilidad de realizar horas extraordinarias dentro de los puestos de trabajo a tiempo parcial, si bien en proporción con la jornada pactada, y siempre que la suma de las horas ordinarias y extraordinarias no supere el límite legal del trabajo a tiempo parcial ${ }^{4}$. Recientemente, sin embargo, la mayor parte de la virtualidad de esta medida ha desaparecido con la aprobación del RD Ley 16/2013 que recoge diferentes medidas para favorecer la contratación estable y mejorar la empleabilidad de los trabajadores y limita esta posibilidad a la realización de horas complementarias.

Por el contrario, persiguiendo el objetivo de dotar a las empresas de mayores posibilidades de flexibilidad interna, en el capítulo III se introducen modificaciones de gran calado en relación con la clasificación profesional, la movilidad funcional y geográfica, la modificación sustancial de las condiciones de trabajo, la suspensión del contrato de trabajo o reducción de la jornada y la negociación colectiva.

En este apartado, la regulación del tiempo de trabajo se modifica mediante la introducción de dos tipos distintos de medidas:

a) La posibilidad de modificar sustancialmente las condiciones de trabajo.

b) La posibilidad de reducir la jornada de trabajo.

Ambos se orientan a incrementar la capacidad de las empresas para modificar el tiempo de trabajo, introduciendo de esta forma importantes instrumentos de flexibilidad interna con el que mejorar la capacidad de adaptación de las empresas desde esta perspectiva.

Tanto la modificación sustancial de las condiciones de trabajo (entre ellas la jornada de trabajo) como la reducción del tiempo de trabajo (cuando se proponga una disminución del tiempo de trabajo de entre un 10 y un 70 por ciento de la jornada de trabajo computada sobre la base de una jornada diaria, semanal, mensual o anual)se podrán producir cuando existan probadas razones económicas, técnicas, organizativas o de producción, definidas por su relación con la competitividad, productividad u organización técnica o de trabajo de la empresa. La prueba de las causas económicas implica la existencia de resultados de la empresa de los cuales se desprenda una situación económica negativa. Por su parte, las razones técnicas, organizativas o de producción implican que se produzcan en la empresa cambios en los ámbitos de los instrumentos de producción, de la organización del

\footnotetext{
${ }^{4}$ Dependiendo del convenio colectivo del sector, generalmente las horas extraordinarias son el $15 \%$ de las horas ordinarias.
} 
trabajo o de la producción, o en la demanda de productos o servicios fabricados por la empresa.

La modificación sustancial de la jornada de trabajo podrá tener un carácter individual o colectivo. Tendrá un carácter colectivo cuando, en un período de noventa días, afecte al menos a:

a) Diez trabajadores, en las empresas que ocupen menos de cien trabajadores.

b) El 10 por ciento del número de trabajadores de la empresa en aquellas que ocupen entre cien y trescientos trabajadores.

c) Treinta trabajadores, en las empresas que ocupen más de trescientos trabajadores.

Por el contrario, se considera de carácter individual la modificación que, en el periodo de referencia establecido, no alcance los umbrales señalados para las modificaciones colectivas.

Las modificaciones deberán ser comunicadas por la empresa con una antelación mínima de 15 días. En el caso de las modificaciones individuales, si el trabajador se sintiese perjudicado, podrá rescindir su contrato, con una indemnización de 20 días de salario por año trabajado, o reclamar ante los tribunales, que podrán declarar la modificación justificada o injustificada. Cuando la modificación revista un carácter colectivo, deberá ir precedida en las empresas en que existan representantes legales de los trabajadores de un período de consultas con los mismos de duración no superior a quince días. Cuando el periodo de consultas finalice con acuerdo se presumirá que concurren las causas justificativas. La decisión sobre la modificación colectiva de las condiciones de trabajo será notificada por el empresario a los trabajadores una vez finalizado el periodo de consultas sin acuerdo y surtirá efectos en el plazo de los siete días siguientes a su notificación, aunque se podrá reclamar en conflicto colectivo

El procedimiento exigido en el caso de la reducción de jornada resulta bastante similar al establecido para las modificaciones sustanciales colectivas:

a) La empresa debe comunicar a la autoridad laboral el inicio del procedimiento, abriendo simultáneamente un proceso de consultas con los representantes legales de los trabajadores.

b) La autoridad laboral trasladará la comunicación empresarial a la entidad gestora de las prestaciones por desempleo y a la Inspección de Trabajo y Seguridad Social. 
c) Cuando finalicen las consultas, el empresario comunicará a los trabajadores y a la autoridad laboral su decisión sobre la reducción de jornada, que surtirá efectos en un plazo de 15 días.

d) Si el proceso finaliza con acuerdo se presumirá que concurren las causas justificativas

e) Contra la decisión empresarial se podrá interponer reclamación ante la jurisdicción social, que declarará la medida justificada o injustificada, que podrá tener el carácter de conflicto social en función del número de trabajadores afectados (10 trabajadores en empresas con menos de 100 trabajadores; el 10 por ciento de los trabajadores en empresas que tengan entre 100 y 300 trabajadores; 30 trabajadores en empresas de más de 300 trabajadores).

En definitiva, a partir de la reforma laboral de 2012 los empresarios en España disponen de una herramienta potencialmente muy poderosa con la incrementar la flexibilidad interna de sus empresas mediante el ajuste y modificación del tiempo de trabajo, y ello por diferentes motivos:

a) Las razones exigidas para el inicio de los procesos de modificación son muy amplias.

b) La reforma suprime la obligación de los empresarios de acreditar la concurrencia de las causas señaladas, obligación que si se contemplaba en la anterior normativa.

c) La iniciativa del proceso corre a cargo de la empresa, sin necesidad de contar con autorización administrativa.

d) Aunque la empresa deberá contar con la opinión de los representantes de los trabajadores, si no se produce acuerdo puede llevar a cabo sus planes de modificación, estableciéndose sobre los trabajadores la carga de la reclamación judicial.

Todo ello otorga una elevada discrecionalidad a la empresa, que resulta especialmente notoria, a nuestro juicio, en el supuesto de la modificación sustancial de la jornada de trabajo con carácter individual. 


\section{La evolución reciente del mercado de trabajo y del empleo a tiempo parcial en España}

El objetivo de este apartado es describir someramente el marco general en el que se inscribe la reforma laboral de 2012 así como introducir alguna primera aproximación de los posibles efectos derivados de la misma, siempre en relación con el empleo a tiempo parcial y específicamente con el empleo a tiempo parcial involuntario a través de una aproximación empírica.

De acuerdo con las cifras de la Encuesta de Población Activa (gráfico 1), el recurso del empleo a tiempo parcial dentro del contexto de destrucción de empleo introducido por la crisis económica ha experimentado un notable crecimiento, desde una tasa de parcialidad del $11,8 \%$ en el primer trimestre de 2008 al $16,41 \%$ en el cuarto trimestre de 2014 (acumulando un crecimiento para el periodo aproximadamente del 36\%). La tasa de parcialidad, tanto total como involuntaria, ha crecido aparentemente de manera constante a lo largo del periodo analizado no observándose importantes cambio en el ritmo de crecimiento antes o después de la reforma laboral. Ello, sin embargo, no quiere decir que la reforma no haya tenido consecuencias, dado que este crecimiento se produce en un contexto de destrucción generalizada de empleo y caída del empleo a tiempo completo. Aún cuando es difícil identificar el efecto ejercido por la reforma laboral sobre el uso del empleo a tiempo parcial con independencia de los efectos derivados de la crisis económica, el gráfico 2 apunta un cambio de tendencia en el ritmo de crecimiento de la tasa de parcialidad, que se venía moderando hasta 2012, para incrementar la magnitud de su crecimiento a partir de ese momento y hasta finales de 2014.

Dicho crecimiento desagregado por género ha sido del $83,4 \%$ para el empleo a tiempo parcial de los hombres (desde el 4,82\% al 7,6\%, tasas de parcialidad masculinas) frente al 16,1\% del empleo a tiempo parcial de las mujeres (desde el $22,5 \%$ al $26,1 \%$, tasas de parcialidad femeninas). Observando de nuevo el gráfico 2 se comprueba como el cambio de tendencia observado en la tasa anual de crecimiento de la tasa de parcialidad ha resultado más acusado en el caso de los hombres.

La involuntariedad que puede encerrar este tipo de empleo se puede definir de múltiples formas. De acuerdo con lo que se denomina como tiempo parcial involuntario "puro" (OCDE, 2010), sería aquel constituido por las personas que no encuentran un trabajo a jornada completa deseando tenerlo. En el primer trimestre de 2008 el 29,5\% del empleo parcial masculino podía de catalogarse como involuntario puro 
frente al 32,4\% para el caso de las mujeres. Sin embargo, en el segundo trimestre del año 2014, ambas cifras se incrementan espectacularmente. Además cambian los roles de género, siendo considerado como involuntario el $68,5 \%$ del empleo parcial para los hombres frente al $60,7 \%$ del empleo parcial de las mujeres.

\section{Gráfico 1: Evolución de la tasa de parcialidad y su involuntariedad por género. Números}
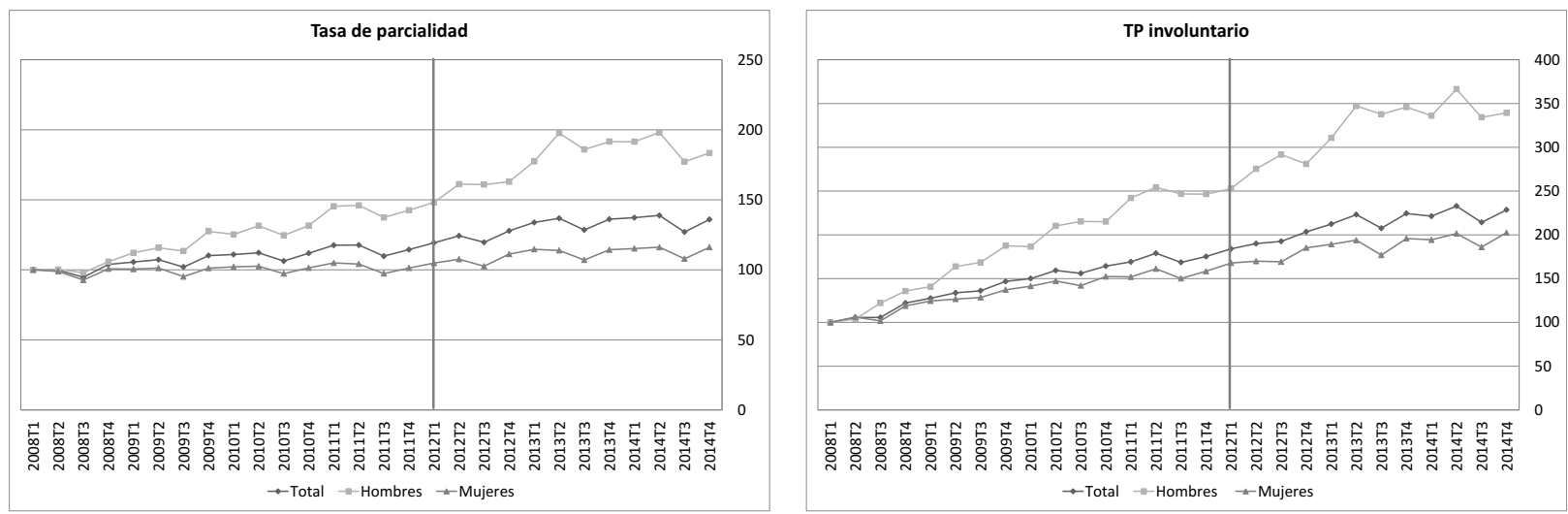

Fuente: EPA, INE.

Gráfico 2: Tasa de variación anual de la tasa de parcialidad por género. Tantos por ciento

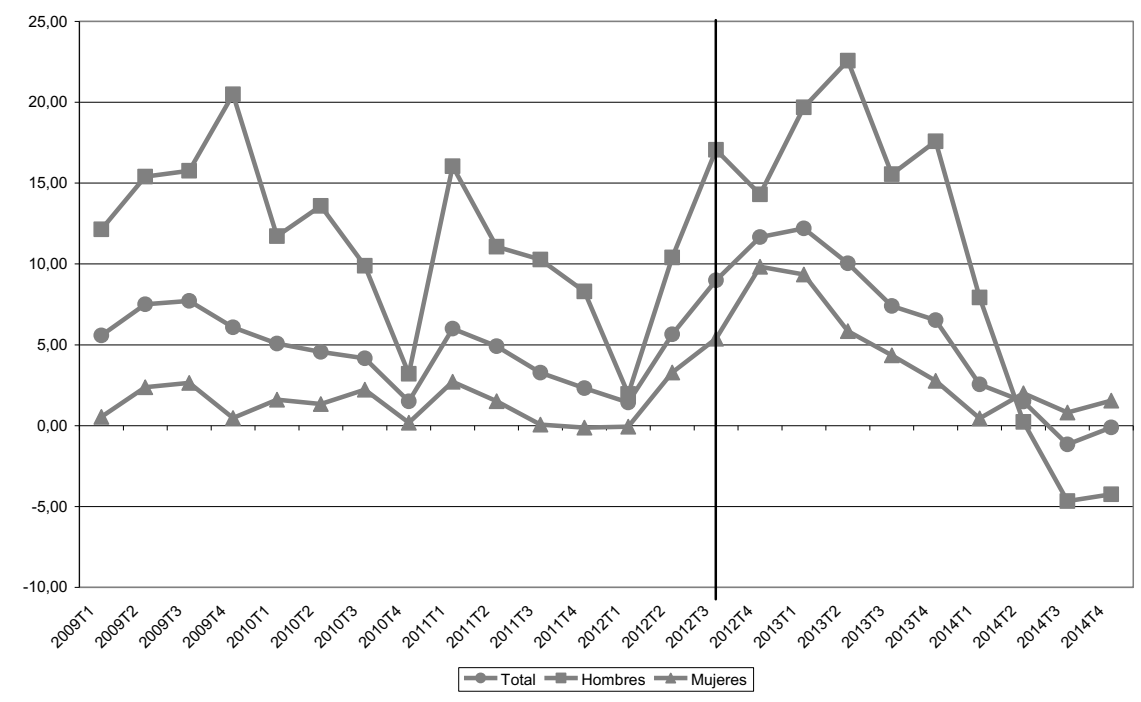

Fuente: EPA, INE. 
El resultado de esta evolución ha sido que la involuntariedad dentro del empleo a tiempo parcial ha acabado siendo mayoritaria para ambos géneros pero sobre todo para los hombres (cuadro 1). Mientras que alrededor de un tercio de los individuos que en el segundo trimestre de 2008 trabajaban a tiempo parcial lo eran de manera involuntaria, esta situación alcanza algo más de dos tercios en el segundo trimestre de 2014.

\section{Cuadro 1: Tiempo parcial involuntario "puro". Porcentaje sobre el total del empleo a tiempo parcial.}

\begin{tabular}{lrrrr}
\hline & $\mathbf{2 0 1 4 T 2}$ & $\mathbf{2 0 1 2 T 2}$ & $\mathbf{2 0 1 0 T 2}$ & 2008T2 \\
\hline Total & $63,4 \%$ & $56,4 \%$ & $49,7 \%$ & $33,6 \%$ \\
Hombres & $69,3 \%$ & $62,6 \%$ & $54,0 \%$ & $30,7 \%$ \\
Mujeres & $61,1 \%$ & $54,4 \%$ & $48,3 \%$ & $34,4 \%$ \\
\hline
\end{tabular}

Fuente: EPA, segundos trimestres.

En definitiva, la naturaleza y características del empleo a tiempo parcial se está modificando de manera muy relevante, tanto desde la perspectiva del género como de las razones que conducen a este tipo de empleo.

El siguiente cuadro 2 recoge información acerca de la tasa de parcialidad y su evolución entre 2008 y 2014 en relación con diferentes colectivos o características. En el segundo trimestre de 2014 (primera columna), el trabajo a tiempo parcial resultaba más frecuente entre los colectivos de: mujeres, jóvenes, personas con situaciones profesionales distintas a las de asalariado o empleador, personas que trabajaban sobre todo en el sector servicios o en el desarrollo de tareas de naturaleza manual y con escasa cualificación. Con el paso del tiempo, en este perfil se observan cambios importantes (última columna), en la medida en que la tasa de parcialidad ha aumentado con una intensidad superior a la registrada para el conjunto de la población para los hombres, que prácticamente ha duplicado su tasa de parcialidad, los más jóvenes pero ahora también para los individuos de edades intermedias, los asalariados del sector privado, la Construcción y ambos extremos de la estructura ocupacional, esto es los trabajadores no manuales más cualificados y los manuales menos cualificados.

Dado nuestro interés en relacionar las medidas consideradas en la reforma laboral de 2012 en relación con la modificación y reducción de la jornada laboral y su posible reflejo con el recurso al empleo a 
tiempo parcial, sobre todo involuntario, seguidamente se recoge información acerca de los cambios acaecidos en el número de horas efectivamente trabajadas tanto por el conjunto de los trabajadores en el empleo principal (gráfico 3) como en el número medio de horas semanales efectivas trabajadas por los trabajadores a tiempo parcial (cuadro 3).

\section{Cuadro 2: Tasa de parcialidad en diferentes colectivos. Porcentajes (*)}

\begin{tabular}{lcccccc}
\hline & $\mathbf{2 0 1 4}$ & $\mathbf{2 0 1 2}$ & $\mathbf{2 0 1 0}$ & $\mathbf{2 0 0 8}$ & $\begin{array}{c}\text { \% Var. } \\
\mathbf{1 4 / 0 8}\end{array}$ & $\begin{array}{c}\mathbf{\%} \text { Var. } \\
\mathbf{1 4 / 1 2}\end{array}$ \\
\hline Total & $\mathbf{1 6 , 4 \%}$ & $\mathbf{1 4 , 7 \%}$ & $\mathbf{1 3 , 2} \%$ & $\mathbf{1 1 , 8 \%}$ & $\mathbf{3 9 , 0} \%$ & $\mathbf{1 1 , 5 6 \%}$ \\
Hombres & $8,2 \%$ & $6,7 \%$ & $5,5 \%$ & $4,2 \%$ & $95,2 \%$ & $22,39 \%$ \\
Mujeres & $26,1 \%$ & $24,2 \%$ & $23,1 \%$ & $22,2 \%$ & $17,6 \%$ & $7,85 \%$ \\
De 16 a 24 años & $41,2 \%$ & $35,8 \%$ & $27,4 \%$ & $18,6 \%$ & $121,5 \%$ & $15,08 \%$ \\
De 25 a 59 años & $14,9 \%$ & $13,4 \%$ & $11,8 \%$ & $10,4 \%$ & $43,3 \%$ & $11,19 \%$ \\
De 60 y más años & $15,8 \%$ & $17,1 \%$ & $15,9 \%$ & $14,8 \%$ & $6,8 \%$ & $-7,60 \%$ \\
Asalariado sector público & $6,7 \%$ & $6,8 \%$ & $7,3 \%$ & $6,3 \%$ & $6,3 \%$ & $-1,47 \%$ \\
Asalariado sector privado & $20,5 \%$ & $17,9 \%$ & $15,6 \%$ & $13,4 \%$ & $53,0 \%$ & $14,53 \%$ \\
Empleador o empresario & $9,5 \%$ & $9,2 \%$ & $8,8 \%$ & $8,7 \%$ & $9,2 \%$ & $3,26 \%$ \\
Otros & $46,1 \%$ & $50,1 \%$ & $46,1 \%$ & $44,8 \%$ & $2,9 \%$ & $-7,98 \%$ \\
Agricultura & $10,4 \%$ & $10,1 \%$ & $8,9 \%$ & $10,1 \%$ & $3,0 \%$ & $2,97 \%$ \\
Industria & $6,1 \%$ & $6,1 \%$ & $4,7 \%$ & $4,9 \%$ & $24,5 \%$ & $0,00 \%$ \\
Construcción & $6,4 \%$ & $5,9 \%$ & $4,4 \%$ & $3,7 \%$ & $73,0 \%$ & $8,47 \%$ \\
Servicios & $19,3 \%$ & $17,3 \%$ & $16,3 \%$ & $15,7 \%$ & $22,9 \%$ & $11,56 \%$ \\
Trabajadores NM-AC & $11,7 \%$ & $9,3 \%$ & & & $25,8 \%$ & $25,81 \%$ \\
Trabajadores NM-BC & $12,4 \%$ & $12,1 \%$ & & & $2,5 \%$ & $2,48 \%$ \\
Trabajadores M-AC & $16,8 \%$ & $15,7 \%$ & & & $7,0 \%$ & $7,01 \%$ \\
Trabajadores M-BC & $25,3 \%$ & $22,0 \%$ & & & $15,0 \%$ & $15,00 \%$ \\
\hline Fuente: EPA, segun & & & & &
\end{tabular}

Fuente: EPA, segundos trimestres.

(*) La tasa de variación de la tasa de parcialidad entre el segundo trimestre de 2014 y el segundo de 2012 fue del 11,56 por ciento. En negrilla los porcentajes superiores a la variación experimentada por la tasa de parcialidad agregada.

Leyenda: NM: No Manuales; M: Manuales; AC: Alta cualificación y BC: Baja Cualificación.

Desde la primera de las perspectivas, los datos ponen claramente de manifiesto un cambio en la jornada laboral realizada por 
la población ocupada. De esta forma, mientras que se observa claramente un incremento en la frecuencia con que los ocupados llevan a cabo jornadas de trabajo menores a 40 horas, se reducen los porcentajes para las jornadas iguales o superiores a la jornada habitualmente relacionada con el contrato a tiempo completo.

Por su parte, la jornada laboral de los trabajadores a tiempo parcial se ha reducido, en términos medios, en 0,4 horas, esto es, un 2,5 por ciento, aunque entre los asalariados a tiempo parcial que trabajan en el sector privado la reducción alcanza casi el 4 por ciento.

\section{Gráfico 3: Horas efectivas trabajadas en la semana del empleo principal}

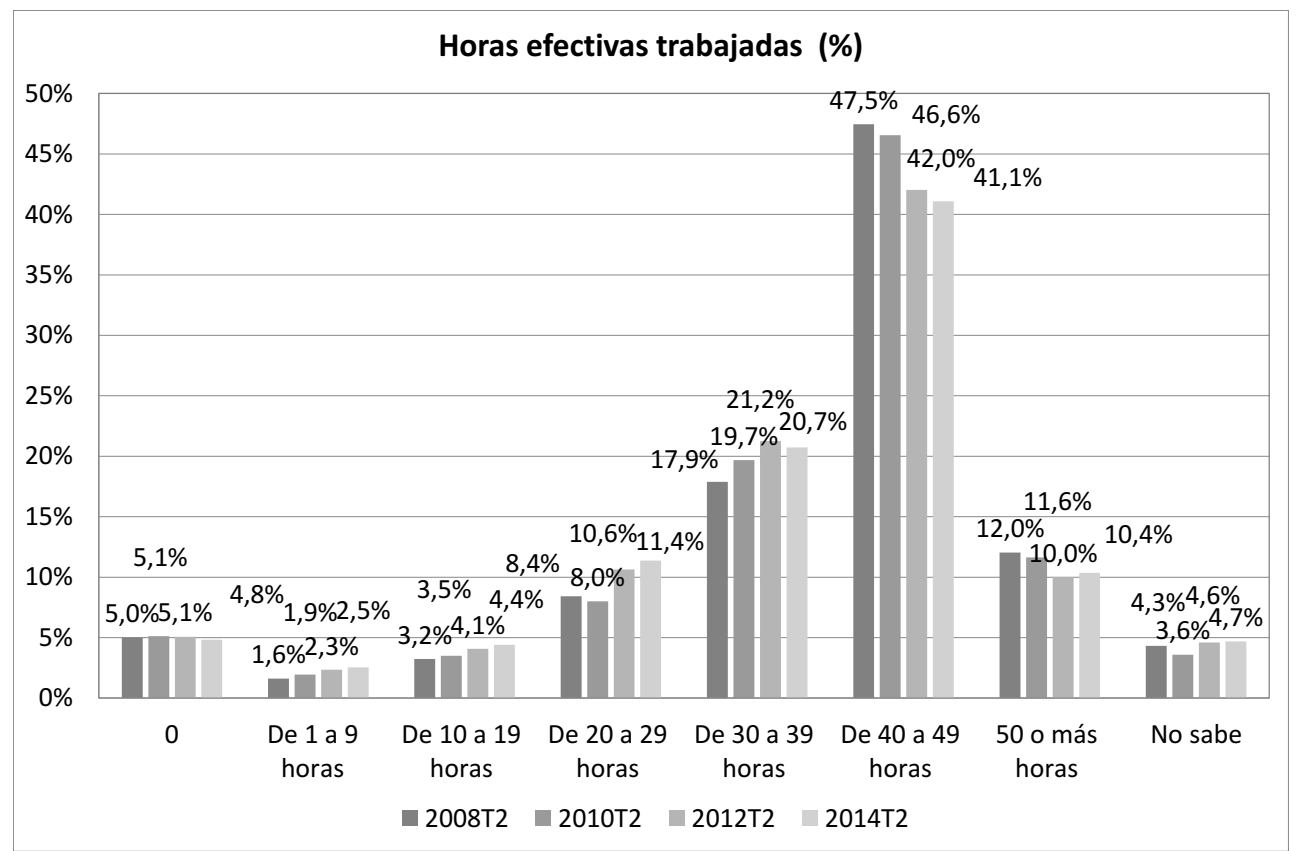

Fuente: EPA, INE.

Para los trabajadores a tiempo parcial el cuadro 4 recoge cómo ha sido la evolución de las horas trabajadas desde diferentes perspectivas. Para todo el periodo de crisis se observa un aumento de las horas no trabajadas frente a una caída de las horas efectivas. Asimismo, tras la reforma laboral, las horas extraordinarias aumentan escasamente entre los trabajadores a TP (0,1 p.p. de diferencia). A falta de un seguimiento temporal más amplio, la posibilidad de aumentar las 
horas extraordinarias entre los trabajadores a TP ha resultado un recurso que se ha utilizado levemente.

Dada la brevedad del tiempo transcurrido desde la aprobación del Decreto-ley 3/2012, se cuenta con poca información con la que conocer la intensidad con la que los nuevos instrumentos de flexibilidad están siendo aplicados en el ámbito de la empresa. Sin embargo, los datos elaborados por el Ministerio de Empleo y Seguridad Social, publicados en la última Encuesta Anual Laboral de 2013 (EAL-2013) aportan algunos datos interesantes al respecto ${ }^{5}$.

\section{Cuadro 3: Número total de horas efectivas semanales por los ocupados a tiempo parcial entre el total de ocupados a tiempo parcial}

\begin{tabular}{lccccc}
\hline & $\mathbf{2 0 1 4}$ & $\mathbf{2 0 1 2}$ & $\mathbf{2 0 1 0}$ & $\mathbf{2 0 0 8}$ & $\mathbf{\% V a r}$ \\
\hline Total & $\mathbf{1 6 , 8}$ & $\mathbf{1 6 , 7}$ & $\mathbf{1 7 , 3}$ & $\mathbf{1 7 , 2}$ & $\mathbf{- 2 , 5}$ \\
Trabajador por cuenta propia & 15,2 & 14,4 & 15,6 & 15,3 & $-0,6$ \\
Empleador & 18,3 & 17,1 & 17,7 & 18,1 & 0,8 \\
Empresario sin asalar. o trabajador indte. & 15,9 & 15,3 & 16,2 & 16,1 & $-1,5$ \\
Miembro de una cooperativa & 23,5 & 12,7 & 25,9 & 19,2 & 22,5 \\
Ayuda en la empresa o negocio familiar & 10,2 & 11,1 & 12,9 & 12,3 & $-16,9$ \\
Asalariados : Total & 17,0 & 17,0 & 17,6 & 17,6 & $-3,2$ \\
Asalariado sector público & 18,1 & 17,1 & 17,7 & 17,4 & 4,3 \\
Asalariado sector privado & 16,9 & 17,0 & 17,5 & 17,6 & $-3,9$ \\
Otra situación & 6,1 & 2,7 & 16,4 & 16,7 & $-63,1$ \\
\hline
\end{tabular}

Fuente: elaboración propia sobre datos de la EPA, segundos trimestres.

\footnotetext{
5 Sobre la metodología de esta encuesta puede consultarse el siguiente enlace, en la página web del INE:

http://www.empleo.gob.es/estadisticas/EAL/welcome.htm

Desafortunadamente la EAL solo ha sido elaborada para el año 2013, no pudiendo realizar comparaciones inter temporales sobre estas cuestiones.
} 


\section{Cuadro 4: Detalles sobre las horas medias trabajadas por los empleados a tiempo parcial. Horas/trabajador(mes)}

\begin{tabular}{lrrrccc}
\hline \multicolumn{1}{c}{$\begin{array}{c}\text { Jornada a tiempo } \\
\text { parcial }\end{array}$} & $\mathbf{2 0 1 4}$ & $\mathbf{2 0 1 2}$ & $\mathbf{2 0 1 0}$ & $\mathbf{2 0 0 8}$ & $\begin{array}{c}\text { Dif. } \\
\mathbf{1 4 / 0 8}\end{array}$ & $\begin{array}{c}\text { Dif. } \\
\mathbf{1 4 / 1 2}\end{array}$ \\
\hline Horas pactadas & 86,60 & 85,9 & 85,4 & 86,6 & 0,0 & $\mathbf{0 , 7}$ \\
Horas pagadas & 86,8 & 86,0 & 85,5 & 86,7 & 0,1 & $\mathbf{0 , 8}$ \\
Horas efectivas & 77,7 & 77,1 & 76,6 & 79,8 & $-2,1$ & $\mathbf{0 , 6}$ \\
Horas no trabajadas & 9,17 & 8,93 & 8,96 & 7,04 & 2,1 & $\mathbf{0 , 2}$ \\
Horas no trabajadas por & 6,26 & 5,96 & 5,63 & 3,46 & 2,8 & $\mathbf{0 , 3}$ \\
$\begin{array}{l}\text { vacaciones y fiestas } \\
\text { Horas no trabajadas por }\end{array}$ & 1,98 & 1,92 & 2,31 & 2,68 & $-0,7$ & $\mathbf{0 , 1}$ \\
$\begin{array}{l}\text { Incapacidad Temporal } \\
\text { Horas extras por }\end{array}$ & 0,26 & 0,18 & 0,18 & 0,2 & 0,1 & $\mathbf{0 , 1}$ \\
trabajador & & & & & & \\
\hline
\end{tabular}

Fuente: EPA, segundos trimestres.

\section{Gráfico 4: Tipos de medidas de flexibilidad internas vinculadas al tiempo de trabajo. Porcentaje sobre el total de empresas en cada categoría $(*)$}

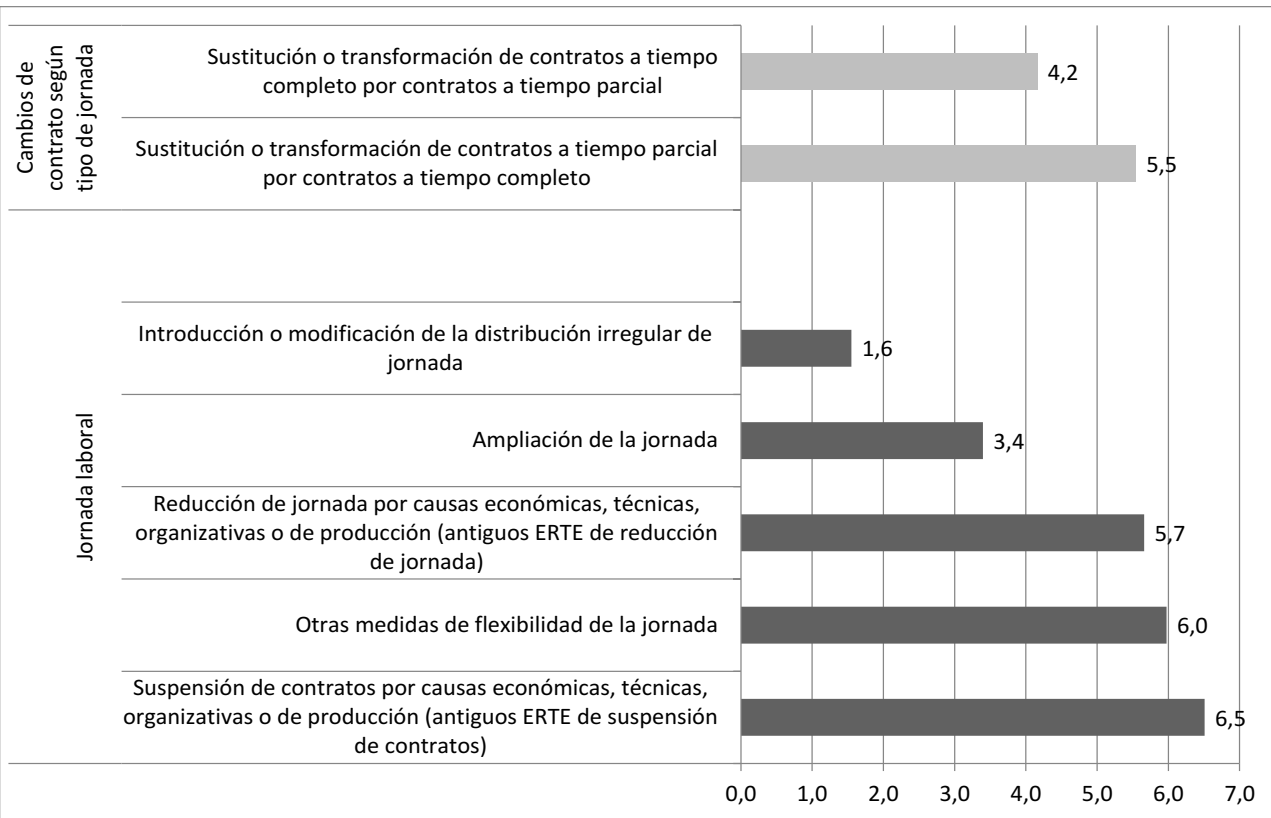

Fuente: Encuesta Anual Laboral, 2013. $\mathrm{M}^{\mathrm{o}}$ de Empleo y SS.

(*) En una empresa se pueden haber aplicado una o más medidas de flexibilidad. 
En relación con la modificación del tiempo de trabajo, en el año 2013 las medidas más frecuentemente aplicadas por las empresas implicaron antes una modificación de la jornada laboral que cambios en los distintos tipos de contratos. De acuerdo con los datos del gráfico 4, mientras que el 4,2 por ciento de las empresas han reducido jornada sustituyendo contratos a tiempo completo por otros a tiempo parcial, el 5,7 por ciento de las empresas han reducido jornada aduciendo los motivos considerados por la reforma, en tanto que el 6 por ciento a emprendido otro tipo de medidas de flexibilidad de la jornada.

Ambos datos, sustitución de trabajo a TC por TP y reducción de la jornada, señalan en la dirección de una reducción del tiempo de trabajo o su uso como mecanismo de ajuste por parte de las empresas, pudiendo ser considerados como indicios con los que cuantificar la intensidad con que las empresas están procediendo a utilizar los instrumentos de flexibilidad puestos a su disposición por la reforma laboral de 2012. Sin embargo, dado que la EAL solo se encuentra disponible para el año 2013, estas tendencias deben tomarse con la necesaria cautela, al menos hasta que se disponga de información para un periodo de tiempo más prolongado.

También es importante destacar como la medida más frecuentemente utilizada por las empresas ha sido la supresión de contratos por razones económicas, técnicas, organizativas o de producción, instrumento utilizado por el 6,5 por ciento de las empresas.

Quizás no resulta sorprendente, dada la redacción de la reforma laboral y la asimetría introducida en el poder relativo de negociación de las dos partes implicadas en la relación laboral, que en todos los instrumentos considerados el acuerdo entre empresa y representantes de los trabajadores resulte ser el procedimiento más frecuente (gráfico 5). En todos los casos los porcentajes de acuerdo superan el 90 por ciento, salvo cuando lo que se plantea es la supresión temporal del contrato de trabajo o cambios irregulares en la jornada laboral.

Por último (gráfico 6), parece claro que las medidas de modificación de la jornada de trabajo resultan más frecuentes cuanto menor es el número de trabajadores de la empresa. En todos los casos, los porcentajes más frecuentes se observan en los casos en que el ajuste afectó a no más del 20 por ciento de las plantillas. Esto es especialmente notorio en los casos en que la modificación implicó cambios en el contrato de trabajo (alrededor del 70 por ciento de los casos afectó a no más del 20 por ciento de los trabajadores). Por el contrario, la incidencia de la modificación de la jornada irregular y de las medidas de reducción de jornada resulta en cierta medida más 
homogéneas en función del porcentaje de la plantilla sobre la que se aplicó.

Se concluye, por tanto, sobre la base de estos análisis descriptivos iniciales, que la reforma laboral de 2012 parece haber resultado efectiva en la consecución de sus objetivos, esto es, dotar a las empresas de un mayor grado de flexibilidad interna, y que en relación con la motivación concreta de este artículo, se manifiesta en la observación de ciertas modificaciones en el tiempo de trabajo y el uso de la contratación a tiempo parcial. No es fácil, sin embargo, saber con exactitud que hubiera sucedido en ausencia de la reforma laboral o que parte de estos cambios se deben solo exclusivamente a la crisis económica. En todo caso, resulta claro que los instrumentos en que se expresan las medidas de flexibilidad interna promovidas por la legislación han sido objeto de un notable uso por parte de las empresas.

\section{Gráfico 5: Porcentaje sobre el total de empresas que han aplicado cada una de las medidas de flexibilidad interna vinculadas al tiempo de trabajo con o sin acuerdo (*)}

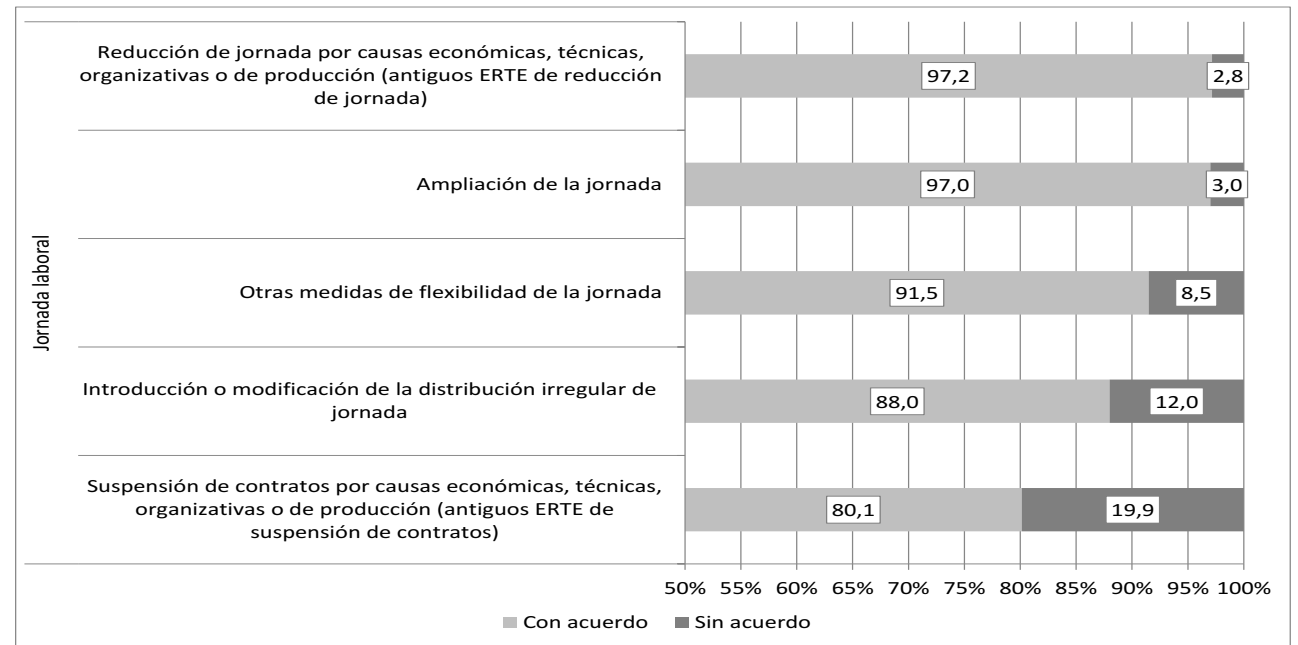

Fuente: Encuesta Anual Laboral, 2013. M de Empleo y SS.

(*) En una empresa se pueden haber aplicado una o más medidas de flexibilidad. 


\section{Gráfico 6: Distribución (\%) de los trabajadores afectados por las diferentes medidas de flexibilidad interna vinculadas al tiempo de trabajo (*)}

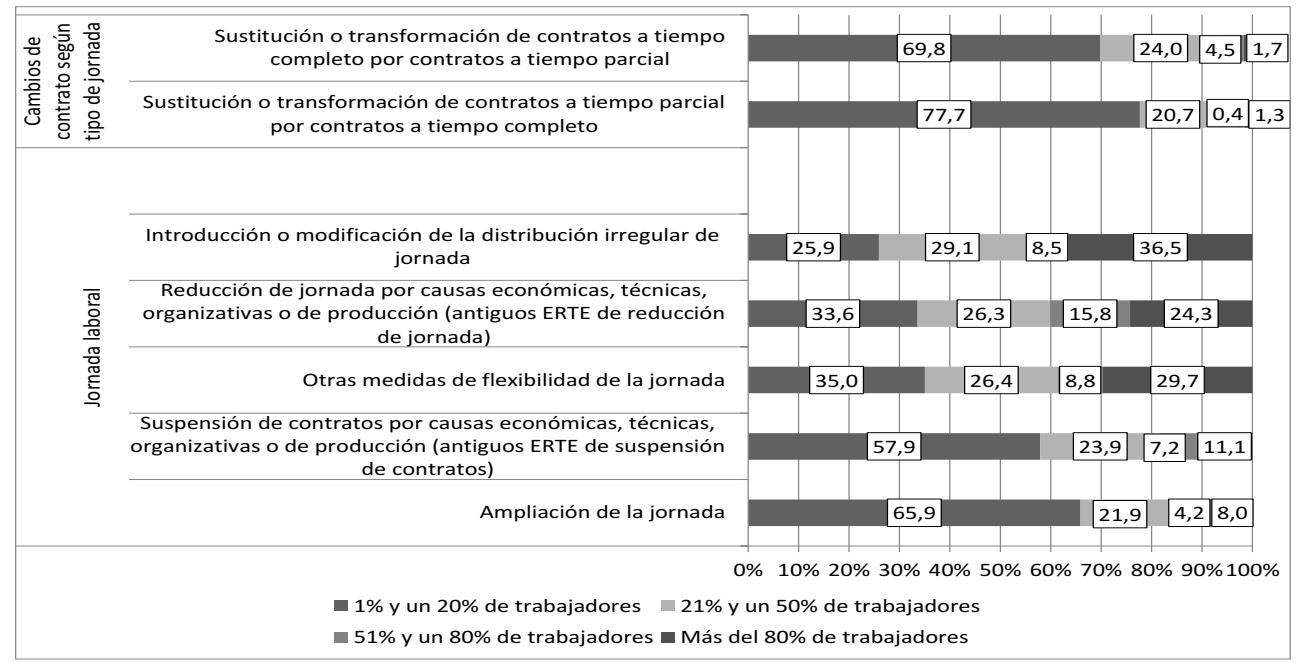

Fuente: Encuesta Anual Laboral, 2013. M $^{\mathrm{o}}$ de Empleo y SS.

(*) En una empresa se pueden haber aplicado una o más medidas de flexibilidad.

\section{Tiempo parcial involuntario, crisis económica y la reforma laboral de 2012}

Dos son hasta ahora los principales resultados que se ha mostrado en este trabajo. En primer lugar, hemos comprobado cómo se ha producido un incremento notable en el uso del empleo a tiempo parcial. En segundo lugar, se observa una modificación en su naturaleza, desde una definición normalizada, vinculada con las decisiones voluntarias de trabajo emprendidas especialmente por las mujeres por motivos de compatibilización de la vida familiar $y$ profesional, hasta otra de carácter involuntario, en la que irrumpe con fuerza la mano de obra masculina pero sobre todo desde patrones asociados a la involuntariedad. Más allá de estos hechos, y aunque los datos descriptivos previos apuntan ciertos indicios sustentando la hipótesis de que la reforma de 2012 ha desempeñado algún papel, quizás notable, en estos cambios, nuestro interés es averiguar el papel que habría desempeñado la reforma laboral de 2012 en estos comportamientos, sobre la base de las importantes posibilidades de flexibilidad interna, especialmente en relación con el tiempo de trabajo, introducidas por la reforma laboral de 2012. 
Para ello, una primera pregunta que deberíamos contestar es el carácter activo o pasivo que presenta el notable y reciente incremento experimentado por el empleo a tiempo parcial en España, especialmente de carácter involuntario. Bajo nuestro punto de vista, el comportamiento observado en el trabajo a tiempo parcial tendría un carácter pasivo si fuera consecuencia de los cambios producidos por la crisis económica sobre la distribución sectorial del empleo. El trabajo a tiempo parcial aumentaría o se reduciría en la medida en que los sectores tradicionalmente más vinculados con esta modalidad contractual viesen aumentar o disminuir el tamaño de su empleo como consecuencia de la crisis económica. Por el contrario, la evolución observada en relación con el tiempo parcial tendría un carácter activo en la medida en que se observase cierta independencia en su comportamiento respecto de los efectos de los procesos de destrucción de empleo inducidos por la crisis. De acuerdo con la primera de las ideas, habría que esperar que fueran los cambios en la distribución sectorial del empleo los que gobernasen la evolución de la parcialidad. Desde la segunda de las perspectivas, deberíamos pensar en algún factor alternativo, los efectos inducidos por la reforma laboral por ejemplo, o las modificaciones productivas introducidas por la propia crisis, para explicar el comportamiento de la contratación a tiempo parcial.

Siendo conscientes de la complejidad de los efectos inducidos por la crisis económica, tanto sobre la distribución sectorial de la destrucción de empleo pero también sobre distintos aspectos de la actividad de las empresas, como la composición de su demanda de trabajo o en general sobre sus políticas de recursos humanos, resulta complicado dotar de un significado claro al segundo componente que identificamos con un comportamiento activo en la evolución del empleo a tiempo parcial en España. De esta forma, el propósito fundamental del análisis shift-share llevado a cabo se centra fundamentalmente en determinar hasta qué punto es posible rechazar la idea de que la evolución de la parcialidad se deba exclusiva y pasivamente a la evolución del empleo sectorial inducida por la crisis económica. En nuestra opinión, sin embargo, el segundo de los componentes nos informará en cierta medida también de si los procesos productivos han cambiado de manera generalizada o solo a cambios en determinados sectores. En la medida que la crisis ha afectado en mayor medida a ciertos sectores y la reforma laboral es igual para todos ellos, se puede inducir, que si la utilización del TP es generalizada su origen debería asociarse en mayor medida con la reforma laboral.

Para responder a esta pregunta vamos a desarrollar, como ya se ha mencionado, un análisis shift-share o de descomposición sobre el 
crecimiento del empleo a tiempo parcial. El objetivo será determinar si su evolución se debe a:

a) Un cambio en la distribución sectorial del empleo, por la destrucción de los sectores mayormente vinculados con el empleo a tiempo completo y/o el crecimiento del empleo en actividades tradicionalmente vinculadas con la parcialidad.

b) O bien, debido a la generalización del empleo a tiempo parcial en todos los sectores lo cual señalaría hacia demostraría la existencia de una pauta de flexibilización interna generalizada.

En la medida en que en los cambios se observan claras pautas por género y se relacionan especialmente con el empleo a tiempo parcial de carácter involuntario, los análisis se desagregarán considerando ambas perspectivas.

El cambio observado en la distribución del empleo en función del tipo de jornada laboral viene expresado por:

$$
\Delta\left(\frac{E_{i}}{E}\right)=\sum_{i=0}^{N} \Delta\left(\frac{E_{i j}}{E_{j}}\right) \frac{\overline{E_{J}}}{E}+\sum_{i=0}^{N}\left(\overline{\frac{E_{l \jmath}}{E_{J}}}\right) \Delta \frac{E_{j}}{E}
$$

siendo $\frac{E_{i}}{E}$ el peso porcentual o presencia del empleo según su jornada laboral $i$ (tiempo completo o parcial) en el empleo total "E"; $\frac{E_{i j}}{E_{j}}$ el peso porcentual del empleo asociado a cada uno de los tipos de jornadas laborales $i$ en una determinada actividad $\mathrm{j}$ sobre el empleo total en dicha actividad "E " $\mathrm{j}_{\mathrm{j}} \frac{E_{j}}{E}$ el peso porcentual del empleo sectorial de cada una de las ramas de actividad $j$ sobre el empleo total "E" ${ }^{6}$. Por consiguiente, el cambio en la parcialidad puede explicarse en función de:

\footnotetext{
${ }^{6}$ Se trata de descomponer los cambios a lo largo del tiempo que se suceden en la matriz de datos resultante de cruzar la información sobre el tipo de jornada y el sector de actividad. El análisis ha considerado información sobre un total de 21 sectores de actividad garantizando que no existan casillas vacías a lo largo del análisis.
} 
a) $\sum_{i=0}^{N} \Delta\left(\frac{E_{i j}}{E_{j}}\right) \frac{\overline{E_{j}}}{E}$; el cambio en la distribución de la jornada laboral dentro de cada actividad económica, lo que se denomina efecto interno. Es decir, si la parcialidad, expresión de la flexibilización en el tiempo de trabajo, se ha incrementado en todos y cada uno de los sectores con independencia del cambio en la distribución sectorial del empleo.

b) $\sum_{i=0}^{N}\left(\frac{\overline{E_{l_{j}}}}{E_{J}}\right) \Delta \frac{E_{j}}{E} ;$ o en relación al cambio sectorial, llamado efecto externo; el cambio generalizado de los sectores analizados, de manera que el aumento del empleo a tiempo parcial estaría fundamentado en el crecimiento, o al menos permanencia, de aquellos sectores donde el tiempo parcial sea superior.

El cuadro 5 recoge los resultados obtenidos en el cálculo del shift-share para el empleo a tiempo parcial en su conjunto, sin considerar su carácter voluntario o involuntario. Los resultados se detallan para mujeres, hombres y ambos géneros. Puede observarse como para el periodo completo y ambos géneros, el 76 por ciento del crecimiento observado en el empleo a tiempo parcial se relaciona con cambios internos ocurridos en la totalidad de los sectores, con independencia del crecimiento o caída del empleo en cada uno de ellos. A pesar de ello, la generalización del empleo a tiempo parcial ha resultado más intensa en sectores tales como el Comercio, la Hostelería y la Educación. El 24 por ciento restante se debe a cambios sectoriales, es decir, a la evolución del empleo en los sectores donde el tiempo completo es predominante. En este caso, destacaría evidentemente la caída del empleo en el sector de la Construcción y durante los últimos años en el sector Educativo. 


\section{Cuadro 5: Descomposición del crecimiento del empleo a tiempo} parcial por género

\begin{tabular}{lccccc}
\hline \multicolumn{2}{c}{ Valor absoluto } & $\begin{array}{c}\text { Var. abs. } \\
\text { peso del TP }\end{array}$ & \multicolumn{2}{c}{$\begin{array}{c}\text { Distribución } \\
\text { (\%) }\end{array}$} \\
\hline \multicolumn{2}{r}{ E. Interno } & E. Externo & $\Delta$ Ei/E & E. Interno & E. Externo \\
Ambos géneros & & & & \\
$2014-2008$ & 0,035 & 0,011 & 0,046 & $76 \%$ & $24 \%$ \\
$2014-2012$ & 0,014 & 0,003 & 0,017 & $81 \%$ & $19 \%$ \\
$2012-2010$ & 0,013 & 0,002 & 0,014 & $89 \%$ & $11 \%$ \\
$2010-2008$ & 0,009 & 0,006 & 0,015 & $59 \%$ & $41 \%$ \\
Hombres & & & & & \\
$2014-2008$ & 0,033 & 0,008 & 0,041 & $80 \%$ & $20 \%$ \\
$2014-2012$ & 0,014 & 0,002 & 0,015 & $90 \%$ & $10 \%$ \\
$2012-2010$ & 0,010 & 0,002 & 0,012 & $84 \%$ & $16 \%$ \\
$2010-2008$ & 0,009 & 0,004 & 0,013 & $69 \%$ & $31 \%$ \\
Mujeres & & & & & $-4 \%$ \\
$2014-2008$ & 0,041 & $-0,002$ & 0,039 & $104 \%$ & $21 \%$ \\
$2014-2012$ & 0,015 & 0,004 & 0,019 & $79 \%$ & $-40 \%$ \\
$2012-2010$ & 0,016 & $-0,004$ & 0,011 & $140 \%$ & $-10 \%$ \\
$2010-2008$ & 0,009 & $-0,001$ & 0,008 & $110 \%$ & \\
\hline
\end{tabular}

Fuente: elaboración propia sobre datos de la EPA, segundos trimestres.

De acuerdo con la cuantificación de los efectos, parece que el recurso al empleo a tiempo parcial se vincula en mayor medida con una generalización de su uso y no con la caída de sectores donde el empleo a tiempo completo es más frecuente. El mismo resultado se produce para mujeres y hombres, aunque las mujeres la modifican, presentando un efecto interno mayor.

$\mathrm{Si}$ atendemos a los resultados desagregados temporalmente, vemos que mientras que efecto interno y externo alcanzan porcentajes explicativos similares durante la primera fase de la crisis (2008-2010), es a partir de 2010, coincidiendo con las dos últimas reformas laborales y el avance de la crisis, cuando el efecto interno se convierte en claro dominador. Ambas pautas no pueden desligarse y estarían detrás de la generalización del TP como manera de flexibilización laboral o de un cambio productivo generalizado. En el caso del empleo femenino, la importancia del efecto interno se reduce al avanzar la crisis, y promulgarse las reformas. Para las mujeres, los periodos 2008-2010 y 2010-2012 si supusieron una pérdida de empleo debido a que la destrucción de empleo inducida por la crisis afectó negativamente a 
sectores tradicionalmente femeninos, fuertemente vinculados con la parcialidad (efecto externo negativo).

El cuadro 6 recoge los resultados del análisis shift-share cuando ahora lo limitamos al empleo a tiempo parcial de carácter involuntario. Ahora los resultados son mucho más claros y generalizados por género. Para el conjunto del empleo y el periodo 2008-2014 la práctica totalidad del cambio observado en el empleo a tiempo parcial con carácter involuntario se explica por cambios internos ocurridos en los sectores de actividad, permaneciendo constante la ponderación de cada uno de ellos sobre el empleo total. Hombres y mujeres reproducen con casi una total exactitud este resultado, que tampoco presenta sino unos mínimos matices cuando el análisis lo desagregamos para cada uno de los tres periodos considerados.

Parece, por tanto, que el empleo a tiempo parcial involuntario no tiene demasiado que ver con la creación y destrucción de empleo asociada a la crisis económica ni con su distribución entre los distintos sectores de actividad. El notable crecimiento experimentado por el empleo a tiempo parcial involuntario tiene antes que ver con procesos que han acaecido en el interior del conjunto de los procesos productivos y de trabajo que conforman la estructura sectorial. No parece que haya sido una forma de canalizar la destrucción de empleo ocurrida en determinados sectores en el contexto de la crisis, sino más bien un cambio en la forma en que las empresas, en relación con el conjunto de los sectores, gestionan sus plantillas. Este resultado constituye, a nuestro juicio, un indicio del potencial papel desempeñado por la reforma laboral de 2012 en la organización del tiempo de trabajo. Sin embargo, este indicio debe estar convenientemente matizado por la dificultad de identificar adecuadamente el componente interno con los efectos inducidos por la reforma independientemente de los efectos ejercidos por la crisis en el interior del conjunto de la estructura productiva. 


Cuadro 6: Descomposición del crecimiento del empleo a tiempo
parcial involuntario por género

Fuente: elaboración propia sobre datos de la EPA, segundos trimestres.

Continuando con esta idea, en el cuadro 7 se muestra cómo se distribuyen los flujos de entrada al empleo a tiempo parcial, es decir, el origen de las personas actualmente empleadas a tiempo parcial. Para elaborar estos datos se ha utilizado los datos proporcionados por la EPA enlazable ${ }^{7}$, enlazando la muestra común en los trimestres consecutivos para observar cuál es la situación de los trabajadores a tiempo parcial.

Con estos datos se concluye como desde el año 2008 hasta 2012 ha aumentado levemente la permanencia en el empleo a tiempo parcial, lo que podría indicar indirectamente un leve aumento de la flexibilidad interna en las empresas mediante el uso del empleo a tiempo parcial como herramienta de flexibilización de la jornada laboral. La transformación del empleo a tiempo completo (TC) hasta tiempo parcial (TP) ha caído desde el $14,5 \%$ al $12,7 \%$. Pero lo más importante es que se

\footnotetext{
7 Los datos se encuentran ponderados acorde con los nuevos pesos poblacionales establecidos desde el Censo de 2011. Asimismo, se pondera según el peso o ponderación de destino, tal como, establece el INE para sus estimaciones. http://www.ine.es/prensanp840.pdf
} 
ha incrementado considerablemente el flujo de entrada desde el desempleo, del 5,9\% al 11,7\%. Asimismo, la entrada desde la inactividad al empleo a TP resulta cada vez menos frecuente. Para el empleo a TP involuntario las pautas son similares si bien la permanencia en este tipo de empleo resulta menor en relación al empleo a TP al completo. Una posible explicación de estas pautas es que se estuviera produciendo algún tipo de proceso de sustitución dentro del empleo de las empresas como forma de aplicar las posibilidades de la reforma laboral, cuestión que debe validarse con el tiempo.

\begin{tabular}{|c|c|c|c|c|}
\hline & $\begin{array}{r}2013 \\
\mathrm{~T} 1-\mathrm{T} 2 \\
\end{array}$ & $\begin{array}{r}2012 \\
\mathrm{~T} 1-\mathrm{T} 2 \\
\end{array}$ & $\begin{array}{r}2010 \\
\text { T1-T2 }\end{array}$ & $\begin{array}{r}2008 \\
\text { T1-T2 }\end{array}$ \\
\hline \multicolumn{5}{|c|}{ Distribución de las entradas al TP } \\
\hline Permanencia en el TP & $70,8 \%$ & $67,3 \%$ & $69,7 \%$ & $69,2 \%$ \\
\hline Entradas al TP desde TC & $12,7 \%$ & $14,6 \%$ & $14,5 \%$ & $14,5 \%$ \\
\hline Entradas al TP desde el Paro & $11,7 \%$ & $11,8 \%$ & $8,7 \%$ & $5,9 \%$ \\
\hline \multirow{2}{*}{$\begin{array}{l}\text { Entradas al TP desde la } \\
\text { Inactividad }\end{array}$} & $4,8 \%$ & $6,3 \%$ & $7,1 \%$ & $10,4 \%$ \\
\hline & $100,0 \%$ & $100,0 \%$ & $100,0 \%$ & $100,0 \%$ \\
\hline \multicolumn{5}{|c|}{ Distribución de las entradas al TPI } \\
\hline Permanencia en el TPI & $57,1 \%$ & $50,1 \%$ & $48,7 \%$ & $41,9 \%$ \\
\hline Entradas al TPI desde TPV & $12,8 \%$ & $14,1 \%$ & $18,4 \%$ & $25,0 \%$ \\
\hline Entradas al TPI desde TC & $10,0 \%$ & $13,3 \%$ & $13,9 \%$ & $15,0 \%$ \\
\hline Entradas al TPI desde el Paro & $16,5 \%$ & $18,1 \%$ & $14,1 \%$ & $11,5 \%$ \\
\hline \multirow{2}{*}{$\begin{array}{l}\text { Entradas al TPI desde la } \\
\text { Inactividad }\end{array}$} & $3,6 \%$ & $4,3 \%$ & $4,9 \%$ & $6,6 \%$ \\
\hline & $100,0 \%$ & $100,0 \%$ & $100,0 \%$ & $100,0 \%$ \\
\hline
\end{tabular}

Fuente: elaboración propia sobre datos de la EPA flujos, segundos trimestres.

Dado que, tal como se ha comprobado previamente, las transformaciones más importantes en el empleo a TP involuntario se relacionan con el género, el cuadro 8 desagrega los flujos de la parte final del cuadro previo (orígenes en el paro o la inactividad) para hombres y mujeres. Las diferencias resultan claras, el $24,7 \%$ de los hombres ocupados a TP se encontraban en desempleo el trimestre anterior mientras que la misma cifra para el caso de las mujeres es del $13 \%$. La pauta es similar para las mujeres, aunque reviste una menor intensidad. 
Para cerrar este apartado, en el cuadro 9 se muestra la distribución interna entre hombres y mujeres dentro de los flujos considerados en el cuadro anterior. A la vista de los datos, debemos tener en cuenta que aunque existe una pauta de transformación en el empleo a TP por género, el empleo a TP tanto voluntario como involuntario sigue siendo mayoritariamente femenino y que los flujos de entrada se nutren mayoritariamente de mujeres aunque cada vez en menor cuantía.

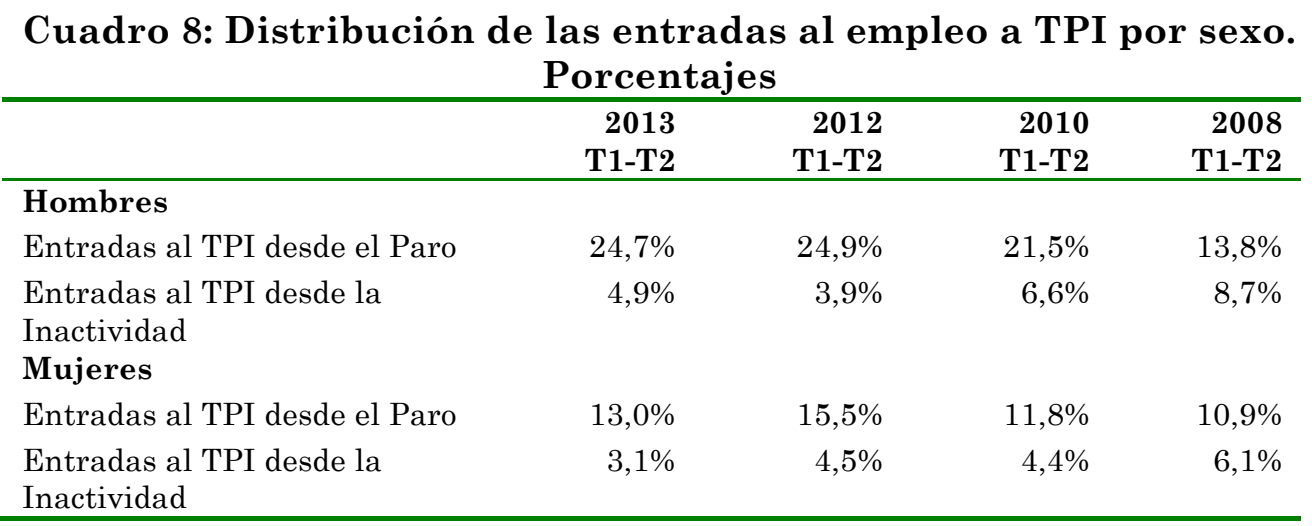

Fuente: elaboración propia sobre datos de la EPA flujos, segundos trimestres

Cuadro 9: Distribución por género de las entradas al empleo a TPI. Porcentajes

\begin{tabular}{lcccc}
\hline & $\mathbf{2 0 1 3}$ & $\mathbf{2 0 1 2}$ & $\mathbf{2 0 1 0}$ & $\mathbf{2 0 0 8}$ \\
& T1-T2 & T1-T2 & T1-T2 & T1-T2 \\
\hline Entradas al TPI desde el Paro & & & & \\
Hombres & $44,3 \%$ & $38,3 \%$ & $37,0 \%$ & $22,9 \%$ \\
Mujeres & $55,7 \%$ & $61,7 \%$ & $63,0 \%$ & $77,1 \%$ \\
Entradas al TP desde la Inactividad & & & \\
Hombres & $40,0 \%$ & $25,3 \%$ & $32,3 \%$ & $25,1 \%$ \\
Mujeres & $60,0 \%$ & $74,7 \%$ & $67,7 \%$ & $74,9 \%$ \\
\hline
\end{tabular}

Fuente: elaboración propia sobre datos de la EPA flujos, segundos trimestres. 


\section{5. ¿De qué depende trabajar a tiempo parcial? Un análisis antes y después de la reforma laboral}

Para completar los análisis llevados a cabo hasta ahora, a continuación se han estimado una serie de modelos de elección discreta sobre la probabilidad de estar ocupado a tiempo parcial frente a otras alternativas de empleo. El objetivo es identificar cuáles son los determinantes de la parcialidad y si estos han cambiado a lo largo del tiempo, quizás en relación con la promulgación y aplicación de la reforma laboral de 2012. Se han estimado un modelo probit y un modelo probit con sesgo de selección (Heckman, 1979) ${ }^{8}$. En un intento de identificar el posible efecto ejercido por la reforma laboral, las estimaciones se han realizado para los años 2011 y 2013, antes y después de la aplicación del Real Decreto-ley 3/2012. Los datos utilizados nuevamente provienen de la EPA enlazable, es decir del segmento de muestra que permanece entre dos trimestres consecutivos (5/6 partes). Entre las variables independientes, además de las más habitualmente señaladas por la literatura, también se ha incluido algunas variables cruzadas, del tipo de contrato poseído en el trimestre anterior y de la jornada laboral en el trimestre anterior ${ }^{9}$. Se trata, por tanto, de analizar de alguna manera también los flujos laborales que conducen al empleo a PT. Por último, todos los modelos se han realizado para el empleo a TP total (cuadro 10) y para el empleo a TP involuntario (cuadro 11).

De acuerdo con el modelo probit para el año 2013 (primeras columnas) el hecho de esta ocupado a tiempo parcial, claramente y tal como es lógico, se encuentra fuertemente determinado por haber estado

\footnotetext{
8 En el filtro de Heckman se ha considerado como variables determinantes la CCAA, la edad por quinquenios y la variable "Mujer", que se trata de tener en cuenta variables que puedan influir en la decisión de participar en el mercado de trabajo pero no en la elección de la duración de la jornada laboral. De este modo se trata de corregir las estimaciones a través del desarrollo de un modelo en dos etapas considerando un sesgo de participación previo a la elección de la jornada laboral. Por otra parte, se intenta evitar que los instrumentos se relacionen con las covariables de las estimaciones principales utilizando variables diferentes para el filtro y el modelo de probabilidad. De no ser así se podría poner en duda la causalidad del modelo, es decir, si las decisiones de participación y parcialidad son independientes (García Ferrer, A., 2009). Por otra parte, debe haber al menos una variable significativa en la ecuación de selección que no aparezca en la ecuación de interés, esencialmente un instrumento válido (Puhani, P., 2000); en nuestro caso, todos los instrumentos resultan significativos.

${ }^{9}$ La CCAA se ha utilizado como variable en la ecuación de selección, con ella, recogemos los efectos regionales.
} 
ocupado a tiempo parcial el trimestre previo, si bien, esta pauta resulta mayor entre los hombres que entre las mujeres (con un coeficiente de 2.961 frente a 2.744). En relación a las transiciones laborales, es muy probable entrar al empleo a TP desde la inactividad y desde el desempleo. Ambas cuestiones resultan mayoritarias entre los hombres. Asimismo, el hecho de ser mujer es un factor muy vinculado con el hecho de trabajar a TP, que incrementa la probabilidad.

Se ha incluido algunas variables que intentan comparar las diferentes decisiones en relación a la parcialidad ante posibles situaciones de cargas familiares. Las variables que combinan el género con el hecho de ser persona de referencia o estar casado ofrecen resultados de signo diferente para hombres y mujeres. Ser persona de referencia o estar casado son situaciones que para las mujeres elevan la probabilidad de estar empleado a TP mientras que para los hombres sucede lo contrario.

Asimismo, algunas situaciones propias del puesto de trabajo como desarrollar un empleo en el sector servicios o un puesto de trabajo temporal son situaciones muy vinculadas con la parcialidad. Paralelamente trabajar en el sector público se encuentra poco relacionado con la parcialidad.

Los modelos probit con sesgo de selección inciden en las mismas pautas generales. A la luz de las primeras estimaciones parece que los hombres mantienen con el empleo a TP una pauta de refugio ante la crisis y acaban en dicha situación tras situaciones de paro e inactividad. Mientras, las mujeres mantienen una fuerte relación con el TP acrecentada por la crisis económica pero también vinculada con su situación familiar y laboral.

¿Eran las pautas anteriores distintas con anterioridad a la reforma laboral y el recrudecimiento de la crisis ${ }^{10}$ ? Se pueden destacar como principales cambios la mayor permanencia actual (2013) de los hombres en el empleo a TP y la mayor vinculación entre parcialidad y temporalidad. Por el contrario, ha caído la probabilidad de entrar al empleo a TP desde un contrato temporal, tanto para hombres como mujeres, y se ha reducido la probabilidad de que una persona con nacionalidad española ocupe un puesto a tiempo parcial frente a una persona con nacionalidad extranjera. Alguno de estos cambios incide en

\footnotetext{
${ }^{10}$ No se incluyen variables coyunturales, de modo, que en la comparación temporal se puede observar tanto el cambio de la reforma como de la crisis. No obstante, se trata de dos probits en dos momentos del tiempo sobre la población ocupada o mayor de 16 años por lo que las variables constantes que mostraría la evolución general de la economía (inflación, paro...) desaparecerían en la estimación.
} 
la utilización del tiempo parcial como herramienta de flexibilización. Por tanto, podrían vincularse con los primeros efectos de la reforma laboral pero también con el recrudecimiento de la crisis o el cambio en la utilización de la parcialidad observado previamente en el análisis del shift-share. En relación con las variables relacionadas con las transiciones laborales, no se observan cambios de importancia.

En el cuadro 11, se han repetido las estimaciones pero limitadas al hecho de ocupar un empleo a TP de manera involuntaria frente al resto de situaciones dentro del empleo. Los fundamentos de este tipo de empleo introducen cambios de importancia. Observando el modelo probit para el año 2013 (primeras columnas), haber estado parado es ahora una de las razones más importantes para ocupar un empleo a TP de manera involuntaria tanto para hombres como para mujeres. Por el contrario, estar en la inactividad presenta ahora un coeficiente negativo. Con menos probabilidad también estarían el hecho de haber estado ocupado de manera temporal o parcial (sea de forma voluntaria o no). También destaca notablemente la pérdida de valor del coeficiente asociado a trabajar a tiempo parcial anteriormente. Los roles de género ante las diferentes situaciones familiares también cambian. Para el TP involuntario la mujer es improbable que está ocupada si es persona de referencia o se encuentra casada. Asimismo, en este caso, el hecho de ser mujer se encuentra poco relacionado con ocupar un empleo a TP involuntario. El modelo probit con sesgo de selección en términos generales ofrece resultados similares, si bien, algunas variables pierden significatividad.

Desde el 2011, los cambios en el empleo a TP involuntario no resultan especialmente notables, manteniéndose las pautas fundamentales. Aumenta notablemente la probabilidad de trabajar a tiempo parcial de manera involuntaria si anteriormente se trabajaba ya a tiempo parcial. Proceder del desempleo continua siendo la situación con una mayor capacidad explicativa de la probabilidad. Se reduce notablemente el tiempo parcial un aumento del mismo entre los hombres que estaban inactivos previamente. La involuntariedad ha caído entre los hombres que previamente eran temporales; estaban parados y se ha hecho más acusada entre los trabajadores del sector público. Muchas de estas pautas se tratan de transformaciones relacionadas con los flujos de entrada al TPI, se podría pensar que la reforma laboral ha incidido en el uso del TP haciendo que en dicha situación laboral incremente su involuntariedad. Si bien, la coexistencia en el tiempo de la reforma laboral con los adversos efectos económicos originados por la duración de una amplia recesión impide extraer conclusiones más firmes. 


\section{Cuadro 10: Probabilidad de trabajar a TP frente a otros} empleos.

\begin{tabular}{|c|c|c|c|c|c|c|c|c|}
\hline \multirow[b]{4}{*}{ Mujer } & \multicolumn{8}{|c|}{2013 (T1-T2) } \\
\hline & \multicolumn{4}{|c|}{ Probit * } & \multicolumn{4}{|c|}{ Probit con sesgo de selección } \\
\hline & \multirow{2}{*}{$\frac{\text { Coef. }}{0,334}$} & \multicolumn{2}{|c|}{$\mathrm{P}>\mathrm{z}$} & \multirow{2}{*}{$\frac{\mathrm{dy} / \mathrm{dx}}{0,033}$} & \multirow{2}{*}{$\frac{\text { Coef. }}{-}$} & \multicolumn{2}{|c|}{$\mathrm{P}>\mathrm{z}$} & \multirow{2}{*}{$\frac{\mathrm{dy} / \mathrm{dx}}{-}$} \\
\hline & & 0,000 & $* * *$ & & & - & - & \\
\hline Estudios Superiores & $-0,010$ & 0,000 & $* * *$ & $-0,001$ & 0,019 & 0,661 & & 0,002 \\
\hline $\begin{array}{l}\text { Nacionalidad española o } \\
\text { doble }\end{array}$ & $-0,137$ & 0,000 & $* * *$ & $-0,014$ & $-0,134$ & 0,019 & $* *$ & $-0,013$ \\
\hline Sector Servicios & 0,566 & 0,000 & $* * *$ & 0,056 & 0,554 & 0,000 & $* * *$ & 0,053 \\
\hline Ocupación Manual & 0,070 & 0,000 & $* * *$ & 0,007 & 0,082 & 0,056 & * & 0,008 \\
\hline Sector Público & $-0,469$ & 0,000 & $* * *$ & $-0,047$ & $-0,461$ & 0,000 & $* * *$ & $-0,044$ \\
\hline Trabajo temporal & 0,424 & 0,000 & $* * *$ & 0,042 & 0,442 & 0,000 & $* * *$ & 0,043 \\
\hline $\begin{array}{l}\text { Mujer x Persona de } \\
\text { Referencia }\end{array}$ & 0,015 & 0,000 & $* * *$ & 0,001 & 0,059 & 0,113 & & 0,006 \\
\hline $\begin{array}{l}\text { Hombre x Persona de } \\
\text { referencia }\end{array}$ & $-0,093$ & 0,000 & $* * *$ & $-0,009$ & $-0,162$ & 0,001 & $* * *$ & $-0,016$ \\
\hline Mujer x Casado & 0,112 & 0,000 & $* * *$ & 0,011 & 0,186 & 0,000 & $* * *$ & 0,018 \\
\hline Hombre x Casado & $-0,129$ & 0,000 & $* * *$ & $-0,013$ & $-0,202$ & 0,000 & $* * *$ & $-0,019$ \\
\hline Mujer x Parado(t-1) & 1,281 & 0,000 & $* * *$ & 0,128 & 1,320 & 0,000 & $* * *$ & 0,127 \\
\hline Hombre x Parado(t-1) & 1,344 & 0,000 & $* * *$ & 0,134 & 1,161 & 0,000 & $* * *$ & 0,112 \\
\hline Mujer x Inactivo(t-1) & 1,272 & 0,000 & $* * *$ & 0,127 & 1,299 & 0,000 & $* * *$ & 0,125 \\
\hline Hombre $\mathrm{x}$ Inactivo(t-1) & 1,560 & 0,000 & $* * *$ & 0,155 & 1,370 & 0,000 & $* * *$ & 0,132 \\
\hline $\begin{array}{l}\text { Mujer x Empleo } \\
\text { temporal(t-1) }\end{array}$ & $-0,132$ & 0,000 & $* * *$ & $-0,013$ & $-0,097$ & 0,231 & & $-0,009$ \\
\hline $\begin{array}{l}\text { Hombre x Empleo } \\
\text { temporal(t-1) }\end{array}$ & $-0,049$ & 0,000 & $* * *$ & $-0,005$ & $-0,153$ & 0,101 & & $-0,015$ \\
\hline $\begin{array}{l}\text { Mujer x Tiempo Parcial(t- } \\
\text { 1) }\end{array}$ & 2,744 & 0,000 & $* * *$ & 0,273 & 2,768 & 0,000 & $* * *$ & 0,267 \\
\hline $\begin{array}{l}\text { Hombre } \mathrm{x} \text { Tiempo } \\
\text { Parcial(t-1) }\end{array}$ & 2,961 & 0,000 & $* * *$ & 0,295 & 2,835 & 0,000 & $* * *$ & 0,273 \\
\hline Constante & $-2,569$ & 0,000 & $* * *$ & - & $-2,408$ & 0,000 & $* * *$ & 0,000 \\
\hline
\end{tabular}


Cuadro 10 (continuación)

\begin{tabular}{|c|c|c|c|c|c|c|c|c|}
\hline \multirow[b]{4}{*}{ Mujer } & \multicolumn{8}{|c|}{2011 (T1-T2) } \\
\hline & \multicolumn{4}{|c|}{ Probit** } & \multicolumn{4}{|c|}{ Probit con sesgo de selección } \\
\hline & \multirow{2}{*}{$\begin{array}{c}\text { Coef. } \\
0,424\end{array}$} & \multicolumn{2}{|c|}{$\mathrm{P}>\mathrm{z}$} & \multirow{2}{*}{$\frac{\mathrm{dy} / \mathrm{dx}}{0,039}$} & \multirow{2}{*}{$\frac{\text { Coef. }}{-}$} & \multicolumn{2}{|c|}{$\mathrm{P}>\mathrm{Z}$} & \multirow{2}{*}{$\frac{\mathrm{dy} / \mathrm{dx}}{-}$} \\
\hline & & 0,000 & $* * *$ & & & - & - & \\
\hline Estudios Superiores & $-0,045$ & 0,000 & $* * *$ & $-0,004$ & $-0,030$ & 0,447 & & $-0,003$ \\
\hline $\begin{array}{l}\text { Nacionalidad española } \\
\text { o doble }\end{array}$ & 0,007 & 0,000 & $* * *$ & 0,001 & $-0,008$ & 0,887 & & $-0,001$ \\
\hline Sector Servicios & 0,543 & 0,000 & $* * *$ & 0,051 & 0,538 & 0,000 & $* * *$ & 0,048 \\
\hline Ocupación Manual & 0,146 & 0,000 & $* * *$ & 0,014 & 0,135 & 0,000 & $* * *$ & 0,012 \\
\hline Sector Público & $-0,440$ & 0,000 & $* * *$ & $-0,041$ & $-0,452$ & 0,000 & $* * *$ & $-0,040$ \\
\hline Trabajo temporal & 0,301 & 0,000 & $* * *$ & 0,028 & 0,321 & 0,000 & $* * *$ & 0,029 \\
\hline $\begin{array}{l}\text { Mujer x Persona de } \\
\text { Referencia }\end{array}$ & $-0,030$ & 0,000 & $* * *$ & $-0,003$ & 0,005 & 0,889 & & 0,000 \\
\hline $\begin{array}{l}\text { Hombre x Persona de } \\
\text { referencia }\end{array}$ & 0,032 & 0,000 & $* * *$ & 0,003 & $-0,086$ & 0,075 & * & $-0,008$ \\
\hline Mujer x Casado & 0,121 & 0,000 & $* * *$ & 0,011 & 0,185 & 0,000 & $* * *$ & 0,016 \\
\hline Hombre x Casado & $-0,175$ & 0,000 & $* * *$ & $-0,016$ & $-0,291$ & 0,000 & $* * *$ & $-0,026$ \\
\hline Mujer x Parado(t-1) & 1,315 & 0,000 & $* * *$ & 0,122 & 1,364 & 0,000 & $* * *$ & 0,121 \\
\hline Hombre x Parado(t-1) & 1,235 & 0,000 & $* * *$ & 0,115 & 1,051 & 0,000 & $* * *$ & 0,093 \\
\hline Mujer x Inactivo(t-1) & 1,322 & 0,000 & $* * *$ & 0,123 & 1,385 & 0,000 & $* * *$ & 0,123 \\
\hline Hombre x Inactivo(t-1) & 1,511 & 0,000 & $* * *$ & 0,141 & 1,305 & 0,000 & $* * *$ & 0,116 \\
\hline $\begin{array}{l}\text { Mujer x Empleo } \\
\text { temporal(t-1) }\end{array}$ & $-0,034$ & 0,000 & $* * *$ & $-0,003$ & 0,017 & 0,824 & & 0,001 \\
\hline $\begin{array}{l}\text { Hombre x Empleo } \\
\text { temporal(t-1) }\end{array}$ & 0,106 & 0,000 & $* * *$ & 0,010 & $-0,031$ & 0,721 & & $-0,003$ \\
\hline $\begin{array}{l}\text { Mujer x Tiempo } \\
\text { Parcial(t-1) }\end{array}$ & 2,757 & 0,000 & $* * *$ & 0,257 & 2,772 & 0,000 & $* * *$ & 0,247 \\
\hline $\begin{array}{l}\text { Hombre x Tiempo } \\
\text { Parcial(t-1) }\end{array}$ & 2,802 & 0,000 & $* * *$ & 0,261 & 2,665 & 0,000 & $* * *$ & 0,237 \\
\hline Constante & $-0,061$ & 0,000 & $* * *$ & - & $-2,557$ & 0,000 & $* * *$ & 0,000 \\
\hline
\end{tabular}

Fuente: elaboración propia. EPA flujos, 2013 (T1-T2) y 2011 (T1-T2).

Nota: Errores robustos (vce(robust)). Average marginal effects (dy/dx). ${ }^{*}$ Pseudo R2: 0,603 ** Pseudo R2: 0,591. Significativo bajo una probabilidad del 99\% (***) 95\% (**) y del 90\% (*). Las variables independientes son dummies donde la categoría de referencia toma en valor 1 y 0 en el resto de casos. Las variables señaladas con (t-1) se refieren al trimestre previo. En la regresión Probit con filtro de selección se consideran en el filtro dummies sobre CCAA, Edad por quinquenios y la variable "Mujer". El filtro resulta significativo en todas las estimaciones consideradas. 
Cuadro 11. Probabilidad de trabajar a TP involuntario frente a otros empleos.

\begin{tabular}{|c|c|c|c|c|c|c|c|c|}
\hline \multirow[b]{4}{*}{ Mujer } & \multicolumn{8}{|c|}{2013 (T1-T2) } \\
\hline & \multicolumn{4}{|c|}{ Probit * } & \multicolumn{4}{|c|}{ Probit con sesgo de selección } \\
\hline & \multirow{2}{*}{$\begin{array}{c}\text { Coef. } \\
0,055\end{array}$} & \multicolumn{2}{|c|}{$\mathrm{P}>\mathrm{z}$} & \multirow{2}{*}{$\frac{\mathrm{dy} / \mathrm{dx}}{0,017}$} & \multirow{2}{*}{$\frac{\text { Coef. }}{-}$} & \multicolumn{2}{|c|}{$\mathrm{P}>\mathrm{z}$} & \multirow{2}{*}{$\frac{\mathrm{dy} / \mathrm{dx}}{-}$} \\
\hline & & 0,000 & $* * *$ & & & - & - & \\
\hline Estudios Superiores & $-0,278$ & 0,000 & $* * *$ & $-0,086$ & $-0,257$ & 0,000 & $* * *$ & $-0,065$ \\
\hline $\begin{array}{l}\text { Nacionalidad española o } \\
\text { doble }\end{array}$ & $-0,228$ & 0,000 & $* * *$ & $-0,070$ & $-0,160$ & 0,035 & $* *$ & $-0,040$ \\
\hline Sector Servicios & 0,323 & 0,000 & $* * *$ & 0,100 & 0,285 & 0,000 & $* * *$ & 0,072 \\
\hline Ocupación Manual & 0,350 & 0,000 & $* * *$ & 0,108 & 0,360 & 0,000 & $* * *$ & 0,091 \\
\hline Sector Público & $-0,192$ & 0,000 & $* * *$ & $-0,059$ & $-0,139$ & 0,047 & $* *$ & $-0,035$ \\
\hline Trabajo temporal & 0,451 & 0,000 & $* * *$ & 0,140 & 0,373 & 0,000 & $* * *$ & 0,094 \\
\hline $\begin{array}{l}\text { Mujer x Persona de } \\
\text { Referencia }\end{array}$ & $-0,057$ & 0,000 & $* * *$ & $-0,018$ & 0,010 & 0,829 & & 0,002 \\
\hline $\begin{array}{l}\text { Hombre x Persona de } \\
\text { referencia }\end{array}$ & $-0,015$ & 0,000 & $* * *$ & $-0,005$ & 0,164 & 0,085 & $*$ & 0,042 \\
\hline Mujer x Casado & $-0,432$ & 0,000 & $* * *$ & $-0,134$ & $-0,287$ & 0,000 & $* * *$ & $-0,073$ \\
\hline Hombre x Casado & 0,059 & 0,000 & $* * *$ & 0,018 & 0,154 & 0,131 & & 0,039 \\
\hline Mujer x Parado(t-1) & 0,802 & 0,000 & $* * *$ & 0,249 & 0,673 & 0,000 & $* * *$ & 0,170 \\
\hline Hombre x Parado(t-1) & 0,756 & 0,000 & $* * *$ & 0,234 & 0,743 & 0,000 & $* * *$ & 0,188 \\
\hline Mujer x Inactivo(t-1) & $-0,106$ & 0,000 & $* * *$ & $-0,033$ & $-0,260$ & 0,047 & $* *$ & $-0,066$ \\
\hline Hombre $\mathrm{x}$ Inactivo(t-1) & $-0,094$ & 0,000 & $* * *$ & $-0,029$ & $-0,154$ & 0,408 & & $-0,039$ \\
\hline $\begin{array}{l}\text { Mujer x Empleo } \\
\text { temporal(t-1) }\end{array}$ & 0,218 & 0,000 & $* * *$ & 0,067 & 0,190 & 0,045 & $* *$ & 0,048 \\
\hline $\begin{array}{l}\text { Hombre x Empleo } \\
\text { temporal(t-1) }\end{array}$ & 0,162 & 0,000 & $* * *$ & 0,050 & 0,126 & 0,313 & & 0,032 \\
\hline $\begin{array}{l}\text { Mujer x Tiempo Parcial(t- } \\
\text { 1) }\end{array}$ & 0,213 & 0,000 & $* * *$ & 0,066 & 0,150 & 0,023 & $* *$ & 0,038 \\
\hline $\begin{array}{l}\text { Hombre } \mathrm{x} \text { Tiempo } \\
\text { Parcial(t-1) }\end{array}$ & 0,223 & 0,000 & $* * *$ & 0,069 & 0,251 & 0,010 & $* *$ & 0,063 \\
\hline Constante & $-0,849$ & 0,000 & $* * *$ & - & 0,618 & 0,006 & $* * *$ & 0,000 \\
\hline
\end{tabular}


Cuadro 11 (continuación)

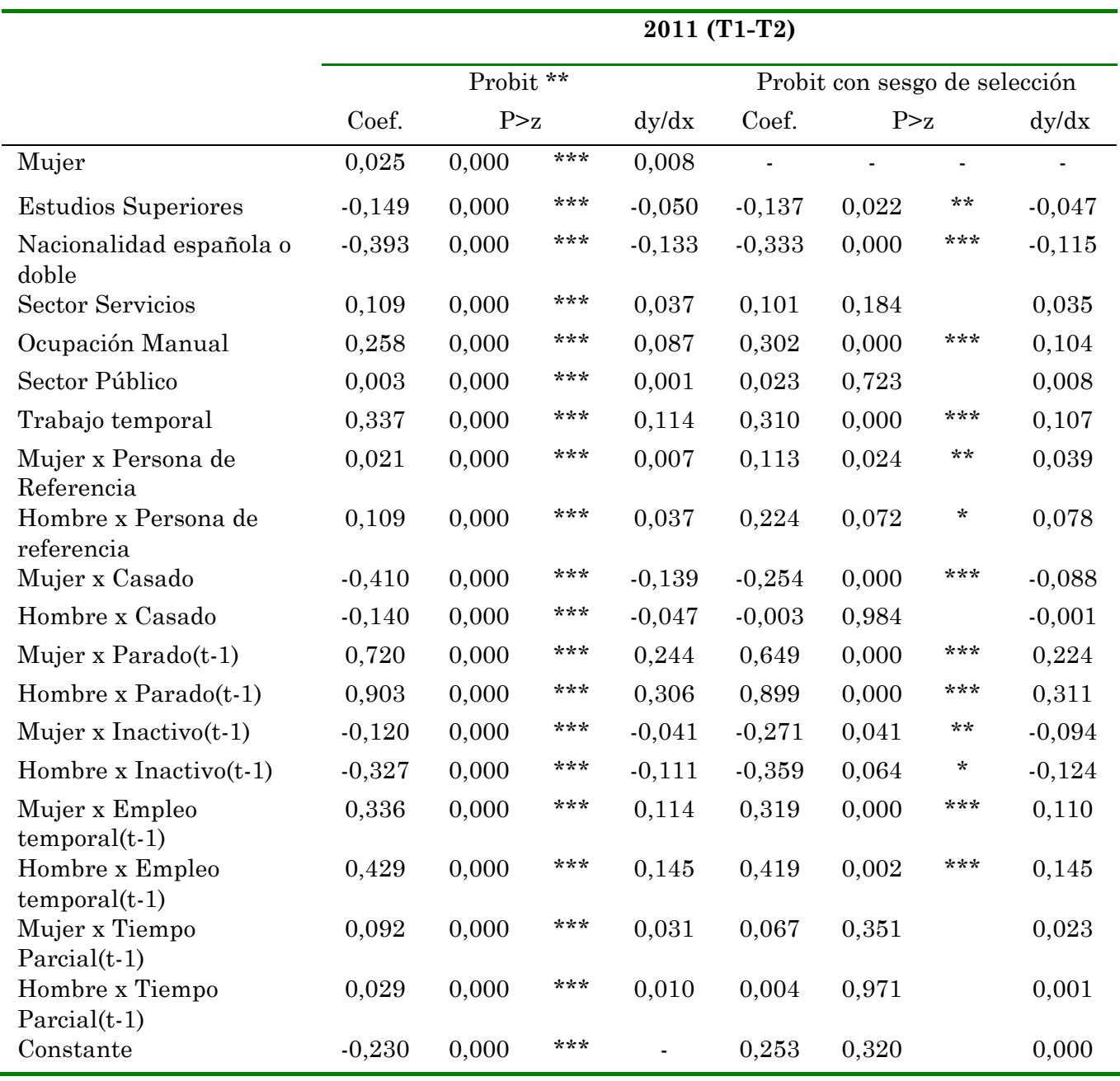

Fuente: elaboración propia EPA flujos, 2013 (T1-T2) y 2011 (T1-T2).

Nota: Errores robustos (vce(robust)). Average marginal effects (dy/dx). ${ }^{*}$ Pseudo R2: 0,155 ** Pseudo R2: 0,129. Significativo bajo una probabilidad del $99 \%(* * *) 95 \%(* *)$ y del $90 \%\left(^{*}\right)$. Las variables independientes son dummies donde la categoría de referencia toma en valor 1 y 0 en el resto de casos. Las variables señaladas con (t-1) se refieren al trimestre previo. En la regresión Probit con filtro de selección se consideran en el filtro dummies sobre CCAA, Edad por quinquenios y la variable "Mujer". El filtro resulta significativo en todas las estimaciones consideradas. 


\section{Conclusiones}

En el contexto de destrucción de empleo inducido por la crisis económica, los datos ponen de manifiesto un crecimiento notable del recurso al empleo a tiempo parcial en nuestro país, que resulta especialmente intenso en el caso del empleo a tiempo parcial de carácter involuntario. Parece que se esté produciendo un cambio en el uso dado al empleo a tiempo parcial, evolucionando desde un modelo de relativa voluntariedad hasta otro centrado en su involuntariedad, desde una utilización vinculada a la compatibilización hasta otra relacionada con el ajuste del mercado de trabajo. Estos cambios tendrían, además, claras implicaciones por género, de forma que se estaría modificando en cierta medida la tradicional definición femenina de este tipo de empleo.

En este contexto, el Real Decreto 3/2012 de medidas urgentes para la reforma del mercado laboral ha introducido importantes modificaciones que facilitan la modificación de las condiciones laborales por parte de la empresa, especialmente en relación con el tiempo de trabajo.

El objetivo del trabajo ha sido evaluar la reforma laboral introducida en nuestro ordenamiento jurídico en 2012 en relación con el tiempo de trabajo, mediante el análisis de la evolución reciente del empleo a tiempo parcial. Nuestra hipótesis es que las modificaciones normativas introducidas estarían propiciando un cambio en el funcionamiento y ajuste del mercado de trabajo español, que se basaría ahora en mayor medida en la modificación del tiempo de trabajo y el uso del empleo a tiempo parcial a través de las posibilidades introducidas en relación con la flexibilidad interna de las empresas.

Los análisis llevados a cabo ponen de manifiesto ciertos resultados. En primer lugar, se observan cambios en el perfil del trabajador a tiempo parcial, junto a un incremento en el uso de este tipo de empleo, a la vez que señalan como se está producido una moderada reducción en el tiempo de trabajo, especialmente en relación con los trabajadores a tiempo parcial. En segundo lugar, también se concluye como el crecimiento del empleo a tiempo parcial no está especialmente vinculado con de los procesos de creación y destrucción de empleo derivados de la crisis económica y su distribución sectorial, especialmente en el caso del tiempo parcial de carácter involuntario. En tercer lugar, el análisis de flujos llevado a cabo señala claramente como la entrada al empleo a tiempo parcial, tanto de carácter voluntario como involuntario, se relaciona sobre todo con entradas desde el desempleo, en tanto que se está produciendo una pérdida de importancia de las 
entradas al tiempo parcial desde el empleo a tiempo completo. Por último, las estimaciones realizadas respecto de la probabilidad de trabajar a tiempo parcial, general o específicamente de carácter involuntario, abundan en la anterior conclusión. La replicación de las estimaciones antes (2011) y después (2013) de la aprobación de la reforma laboral no permite afirmar que se hayan producido cambios significativos, aunque es evidente que el tiempo transcurrido no es probablemente suficiente para llevar a cabo esta evaluación.

En nuestra opinión, estos resultados, no modificación de los determinantes del empleo a tiempo parcial junto a que las entradas a este tipo de empleo se encuentran preferentemente en el desempleo, se constituyen en indicios que cuestionan en cierta medida la posible importancia detentada por la reforma. Del contenido de la reforma laboral se esperaría que el empleo a tiempo parcial encontrara progresivamente su determinación especialmente dentro de la empresa, al posibilitar sus contenidos un desplazamiento de la importancia del ajuste desde el exterior hasta el interior de las empresas. Sin embargo, parece ser que la crisis económica, la destrucción de empleo que conlleva y los intentos de salir del desempleo llevados a cabo por los trabajadores desempleados antes que la modificación del tiempo de trabajo de los ya empleados, continúan siendo los determinantes de mayor calado del empleo a tiempo parcial, también, y quizás con mayor claridad, en el caso del empleo a tiempo parcial de carácter involuntario.

Por tanto, no es posible concluir que la reforma haya ejercido algún tipo de efecto en este sentido, aportando un nuevo mecanismo de ajuste al mercado de trabajo esta vez basado antes en el tiempo de trabajo que en el ajuste de la cantidad de empleo, incentivando la flexibilidad interna sobre la flexibilidad externa.

En definitiva, el aumento del TP en nuestro país y sobre todo su involuntariedad, principalmente en el colectivo masculino, habría venido determinada principalmente por el recrudecimiento de la crisis económico y sus consecuencias. La reforma laboral ha propiciado un marco adecuado para el aumento de la parcialidad pero posiblemente la transformación productiva o flexibilidad interna ya se venía produciendo con anterioridad. Para analizar si en el mercado de trabajo español se ha producido una sustitución de trabajo a tiempo completo por tiempo parcial dentro de las plantillas de las empresas serían necesarios desarrollar análisis desde el lado de la empresa y un estudio más detallado sobre el total de horas trabajadas.

En nuestra opinión, ¿esto es positivo o negativo? La idea de dotar a las empresas de mayores posibilidades de ajuste interno como 
forma de limitar el recurso al ajuste externo nos parece muy interesante, por lo que habría que situar su no consecución en el "debe" del balance de la reforma laboral. Por otra parte, si pensáramos que la reforma laboral introducida en relación a las posibilidades de las empresas de modificar las condiciones sustanciales de la relación laboral resulta excesiva, asimétrica y discrecional, la ausencia de

efectos asociados a su promulgación, al menos desde la perspectiva del tiempo de trabajo, podría considerarse como positiva, en la medida en que permitirá al legislador una nueva oportunidad en el diseño de la reforma, esta vez sobre la base del acuerdo y el diálogo entre las partes, como forma de garantizar probablemente una mayor efectividad.

\section{Bibliografía}

Buddelmeyer, H., Mourre, G. y Ward, M. (2008), "Why Do Europeans Work Part-time? A cross-country panel Analysis", European Central Bank Working Paper, No. 872.

Cam (2012), "Involuntary part-time workers in Britain: evidence from the labour force Surrey", Industrial Relations Journal, 43(3), 242-259.

Canon, M., Kudlyak, M. y Reed, M. (2014), "Is Involuntary Part-time Employment Different after the Great Recession?", The Regional Economist, Julio.

De Pedraza, P., Muñoz, R. y Villacampa, A. (2010), "Determinantes de la situación laboral en España: trabajar a tiempo parcial frente a otras situaciones laborales", Cuadernos de Economía, 33(92), 71-104.

Fagan, C., Warren, T. y McAllister, I. (2001), Gender, employment and working time preferences in Europe, Eurofound, Office for Official Publications of the European Communities, Luxembourg.

García Ferrer, A. (2009), "Causalidad y Econometría”, en J.C. GarcíaBermejo Ochoa (ed.), Sobre la economía y sus métodos, Editorial Trotta.

Heckman J. (1979), "Sample selection bias as a specification error", Econometrica, 47, 153-161. 
Kjeldstad, R. y Nymoen, E. H. (2009), "Part-time work, underemployment and gender", Discussion Paper Statistics Norway Research Department, No. 602.

Kjeldstad, R. y Nymoen, E. H. (2012), "Part-time work and gender: Worker versus job explanations", International Labour Review, 151(12).

OECD (2010), Employment Outlook: Moving Beyond the Jobs Crisis, Geneva.

O'Reilly, J. (1996), "Labour Adjustments throught Part-Time Work", en G. Schmid, J. O'Reilly y K. Schömann (eds.), International Handbook of Labour Market Policy and Evaluation, Edward Elgar.

Puhani, P. (2000), "The Heckman Correction for sample selection and its critique", Journal of Economic Surveys, 14(1), 53-68.

Petrongolo, B. (2004), "Gender Segregation in Employment Contracts", CEPR Discussion Paper, No. 637.

Rubery, J., Fagan, C. y Maier, F. (1996), "Occupational Segregation, Discrimination and Equal Opportunity", en G. Schmid, J. O'Reilly y K. Schömann (eds.), International Handbook of Labour Market Policy and Evaluation, Edward Elgar.

Valleta, R. y Bengali, L. (2013), "What's Behind the Increase in PartTime Work?, Federal Reserve Bank of San Francisco Economic Letter, No. 24. 